\title{
Using model-independent lower bounds to improve pricing of Asian style options in Lévy markets
}

\author{
Griselda Deelstra§, Grégory Rayée§, Steven Vanduffel† and Jing Yao† \\ $\S$ Department of Mathematics, Université Libre de Bruxelles \\ $\dagger$ Department of Economics, Vrije Universiteit Brussel. \\ January 16, 2014
}

\begin{abstract}
Albrecher et al. (2008) have proposed model-independent lower bounds for arithmetic Asian options. In this paper we provide an alternative and more elementary derivation of their results. We use the bounds as control variates to develop a simple Monte Carlo method for pricing contracts with Asian style features. The conditioning idea that is inherent in our approach also inspires us to propose a new semianalytic pricing approach. We compare both approaches and conclude that they both have their merits and are useful in practice. In particular, we point out that our newly proposed Monte Carlo method allows to deal with Asian style products that appear in insurance (e.g. unit linked contracts) in a very efficient way, and outperforms other known Monte Carlo methods that are based on control variates.
\end{abstract}

Keywords: Asian style options, conditional expectation, control variates, stochastic clock.

\section{Introduction}

An Asian option is a derivative whose pay-off is based on the average of the trajectory of an underlying primitive asset. Such smoothing is often regarded as a desired protection against unexpected and brutal changes in the value of the underlying ${ }^{1}$. The same averaging feature also appears naturally when compounding or discounting cash flows, and hence Asian-style payoffs crop up in finance and actuarial science naturally. Among others we mention unit linked products (see e.g. Schrager and Pelsser (2004)), ratchet equity indexed annuities (with Asian-end design) (see e.g. Ballotta (2010) or Lin and Tan (2003)) and variable annuities with flexible premiums (see e.g. Milevsky and Posner (2003)). Technically speaking Asian options also

\footnotetext{
${ }^{1}$ In contrast with standard calls they are less sensitive to speculators who could drive up the gains from the option by manipulating the price of the underlying asset near maturity
} 
appear in other disciplines such as physics (see e.g. Romeo et al. (2003)) and engineering (see e.g. Fenton (1960)). Unfortunately the averaging and thus the path-dependence involved makes options with Asian-style features much harder to value than their plain vanilla counterparts, and this even under the standard Black-Scholes model for the financial market. Hence there has always been a great interest of the academic community in Asian options; see e.g. Boyle and Potapchik (2008) for an overview. They also exist in different flavors but in this paper we focus on the discretely monitored fixed strike Asian option with arithmetic averaging.

We make several contributions in this paper. Our first contribution is that we derive model-independent lower bounds for Asian options valid in a rather general market setting. Model-independent bounds are important because they are solely implied by information available in the market and hence are not prone to model error nor incompleteness of financial markets. The bounds we obtain are closely tied to the ones appearing in Albrecher et al. (2008), but are here obtained in a more straight-forward way. Our approach does not make use of ad-hoc probabilistic arguments but relies on rather simple conditioning techniques combined with well established actuarial theory on stop-loss bounds. This result is also appealing as similar conditioning arguments can be useful to derive bounds for other options. In this paper, we show that the idea of conditioning is also crucial in proposing a new semi-analytic approach.

Our second contribution is that, in the context of a subordinated Brownian motion for modelling the stock returns, we present a semi-analytic pricing method using a two-step approach. The idea of this method is that in a first step we condition on the stochastic clock which makes the nature of the problem multi-variate normal and allows us to make use of very accurate closed form approximations that we apply conditionally. Next, we use Monte-Carlo simulation to account for the stochasticity of the clock itself. This method is astonishing fast and provides tight lower bound approximations. The budget for carrying out simulations is important when economic (real-world) scenarios interplay with (risk neutral) pricing, a situation which appears when assessing the risk of a portfolio of financial instruments over a given horizon (e.g. one year). In these instances this method appears to provide an attractive trade-off between accuracy and efficiency.

Monte-Carlo simulation is a classical approach to value derivatives. It combines flexibility with accuracy, i.e. asymptotically the simulated value converges to the true value. The drawback is that the variance on the estimate decreases slowly which creates room for potentially attractive improvements. Our third contribution is that we show how the bounds that we derive can also be used as so-called control variates to increase the efficiency 
of traditional Monte-Carlo schemes. We compare this method with other promising control variate approaches that were recently proposed by Dingec and Hormann (2012) and by Fusai and Meucci (2008). Several numerical experiments allow us to conclude that our approach is outperforming when pricing insurance contracts with Asian style features such as unit linked contracts or variable annuities with flexible premiums. In contrast, the method of Fusai and Meucci (2008) is better adapted when pricing contracts with a shorter maturity.

The structure of the paper is as follows. In Section 2 we derive modelindependent lower bounds that were introduced in Albrecher et al. (2008) in a more straightforward way. In Section 3 we discuss the use of these bounds as control variates in Monte-Carlo simulations. In Section 4 we introduce the market setting that we focus on, namely a subordinated Brownian motion framework with as particular examples the Variance Gamma and the Normal Inverse Gaussian models. Section 5 is devoted to the derivation of analytical expressions for the lower bounds that we use as control variates in the setting of the Variance Gamma and the Normal Inverse Gaussian models. Section 6 presents a second approach for deriving precise and efficient estimations for the price of an Asian option. Several sets of numerical results are discussed in Section 7. Section 8 provides some extensions, in particular ratchet equity-indexed annuities and unit-linked insurances are discussed. Section 9 concludes the paper.

\section{Model-Independent Bounds for Asian Options}

The bounds we propose to price Asian options efficiently are rooted in actuarial theory on stop-loss order. We first define this ordering concept and provide a modest accompanying result that we further need in the paper. Next we derive three model-independent lower bounds for Asian options. These can also be found in Albrecher et al. (2008) but are here derived in a more straightforward way.

\section{$2.1 \quad$ Stop-loss Bounds}

Stop-loss order is a well established concept in the actuarial literature. It can be formally defined as follows.

Definition 1 (stop-loss order) Let $X$ and $Y$ be two random variables. We say that the random variable ${ }^{2} X$ is smaller than $Y$ in stop-loss order, denoted as $X \leq_{s l} Y$, if for all $d \in \mathbb{R}$ it holds that $E[\operatorname{Max}(X-d, 0)] \leq$ $E[\operatorname{Max}(Y-d, 0)]$.

\footnotetext{
${ }^{2}$ In the remainder of the paper all expectations are tacitly assumed to exist.
} 
It is well known that $\left.X \leq_{s l} Y \Leftrightarrow E[v(X))\right] \leq E[v(Y)]$ for all increasing convex functions $v$, which explains why stop-loss order also appears in the literature under the name increasing convex order. ${ }^{3} \mathrm{~A}$ well-known stop-loss lower bound for $X$ is obtained by conditioning, i.e. for any random variable $Z$ it holds that,

$$
\mathrm{E}[X \mid Z] \leq_{s l} X,
$$

where $Z$ can be any random variable. This result is also known as Jensen's inequality and is instrumental in this paper. A sufficient condition for $X \leq_{s l}$ $Y$ is that $E[X] \leq E[Y]$ and their respective distribution functions cross only once (i.e. there exist a real $c$ so that $F_{X}(x) \leq F_{Y}(x)$ for $x \leq c$ and $F_{X}(x) \geq F_{Y}(x)$ for $\left.x \geq c\right)$. See also Müller and Stoyan (2002) for example. It is then also clear that when $g$ and $h$ are non-decreasing,

$$
g(X)+E[h(X)] \leq_{s l} g(X)+h(X) .
$$

This last inequality will also be often used throughout the paper.

\subsection{Asian options}

In this paper we use the set-up and the notation from Albrecher et al. (2008). Hence we consider an arbitrage-free financial market containing a risky asset with price process $\left\{S_{t}, t \geq 0\right\}$ and a risk-free asset yielding the continuously compounded risk-free rate $r$. Let $\mathbb{P}$ denote the physical probability measure. We consider the arithmetic Asian option with payoff at maturity $T>0$ given by

$$
\operatorname{Max}\left(H_{T}-K, 0\right) .
$$

Here

$$
H_{T}=\frac{1}{n} \sum_{i=1}^{n} S_{t_{i}}
$$

$K$ is the strike and the $t_{i}(i=1,2, \ldots, n)$ are the $n$ monitoring times. Without loss of generality we assume that $t_{n}=T$. The absence of arbitrage opportunities implies there is an equivalent martingale (also called risk neutral) measure $\mathbb{Q}$ such that

$$
E\left[S_{s} \mid S_{t}\right]=S_{t} e^{r(s-t)} \quad s \geq t \geq 0,
$$

holds, where the expectation is taken with respect to $\mathbb{Q}$. Unless otherwise mentioned all expectations appearing in this paper are $\mathbb{Q}$-expectations. The arbitrage-free value of the Asian option at $t=0$ is denoted by $A C(K, n)$ and is determined by

$$
A C(K, n)=e^{-r T} \mathrm{E}\left[\operatorname{Max}\left(H_{T}-K, 0\right)\right] .
$$

\footnotetext{
${ }^{3}$ For more details on properties of stop-loss order and its applications in actuarial science we refer to Shaked and Shanthikumar (1994) or Kaas et al. (2008), amongst others.
} 
It is well known that absence of arbitrage is not sufficient to determine the risk neutral measure $\mathbb{Q}$ uniquely, hence the pricing of an Asian option (and other derivatives) might not be unambiguous. Moreover for any possible choice for the pricing measure $\mathbb{Q}$ the evaluation of (4) is by no means trivial and analytical solutions appear to be out of reach in general. Several methods have then been proposed in the literature including (pseudo) Monte-Carlo simulations, moment-matching techniques and Fast Fourier Transforms. Unfortunately, the successful application of these methods require a further specification of the price process $\left\{S_{t}, t \geq 0\right\}$.

The uncertainty inherent with arbitrage-free pricing then gives an incentive to develop model-independent lower bounds, i.e. bounds for $A C(K, n)$ which are (as much as possible) based on available market information only and do not require modelling assumptions. Hence, in the next section we aim at finding lower bounds for $A C(K, n)$ that are "as close as possible" to $A C(K, n)$ while also "as model free as possible" in the sense that they intend to hold irrespective of the choices one makes for the risk neutral measure and the price process of the risky asset. Indeed, the bounds we propose only require knowledge of prices of plain vanilla call prices essentially. Let us now introduce random variables $H_{T}^{l, t}$ defined as

$$
H_{T}^{l, t}=E\left[H_{T} \mid S_{t}\right] \quad 0 \leq t \leq T .
$$

Using the notation

$$
j(t)=\operatorname{Min}\left\{i \mid t_{i} \geq t\right\},
$$

we find using condition (3) that $H_{T}^{l, t}$ can also be expressed as

$$
H_{T}^{l, t}=A_{T}+\frac{1}{n} \sum_{i=j(t)}^{n} S_{t} e^{r\left(t_{i}-t\right)}
$$

where $A_{T}$ is given as

$$
A_{T}=\sum_{i=1}^{j(t)-1} \frac{1}{n} E\left[S_{t_{i}} \mid S_{t}\right],
$$

and where it is tacitly assumed that $\sum_{i=1}^{j(t)-1}=0$ when $j(t)=1$. Note that Jensen's inequality (1) implies that

$$
H_{T}^{l, t} \leq_{s l} H_{T}
$$

which shows, as per definition of stop-loss order, that the $H_{T}^{l, t}$ might be useful to determine lower bounds for the Asian call price $A C(K, n)$ and this is the topic of the next section. 


\subsection{Albrecher's et al. model-independent lower bounds}

\subsubsection{First lower bound}

Let us consider the case $0 \leq t \leq t_{1}$. In this instance $H_{T}^{l, t}$ clearly writes as,

$$
H_{T}^{l, t}=\frac{1}{n} \sum_{i=1}^{n} S_{t} e^{r\left(t_{i}-t\right)}
$$

Let us observe that

$$
H_{T}^{l, t}=E\left[H_{T}^{l, t_{1}} \mid S_{t}\right] .
$$

In particular this implies that for all $t \in\left[0, t_{1}\right], H_{T}^{l, t} \leq_{s l} H_{T}^{l, t_{1}}$ so that the best lower bound for the Asian call price $A C(K, n)$, based on $H_{T}^{l, t}$, is reached when $t=t_{1}$. For its value, denoted by $L B_{1}$, we find that

$$
\begin{aligned}
& L B_{1}=e^{-r T} \mathrm{E}\left[\operatorname{Max}\left(H_{T}^{l, t_{1}}-K, 0\right)\right] \\
& =\frac{e^{-r T}}{n} \mathrm{E}\left[\operatorname{Max}\left(S_{t_{1}}-\frac{n K}{\sum_{i=1}^{n} e^{r\left(t_{i}-t_{1}\right)}}, 0\right)\right] \sum_{i=1}^{n} e^{r\left(t_{i}-t_{1}\right)} \\
& =\frac{1}{n} C\left(\frac{n K}{\sum_{i=1}^{n} e^{r\left(t_{i}-t_{1}\right)}}, t_{1}\right) \sum_{i=1}^{n} e^{-r\left(T-t_{i}\right)} \text {, }
\end{aligned}
$$

where in the last step we have tacitly introduced the notation $C(K, t)$ to denote the value of a standard European Call with strike $K$ and maturity $t>0$. In summary we have that

$$
L B_{1} \leq A C(K, n) .
$$

and only the non-arbitrage assumption was needed to derive the expression for $L B_{1}$. Hence the value for $L B_{1}$ can be observed in the market as soon as the European calls are quoted.

We remark that expression (6) was already derived in Albrecher et al (p. 127 eq. (4), 2008). They resorted to comonotonicity theory in order to do so but this is unnecessary. In the next section we sharpen the bounds further. However this goes at the cost of imposing some more structure on the market.

\subsubsection{Second lower bound}

The first lower bound might not be sharp because the variable $H_{T}^{l, t_{1}}$, obtained by taking the conditional expectation of $H_{T}$ with respect to $S_{t_{1}}$, may not always depict the best possible approximation for $H_{T}$ (which incorporates the randomness of $S_{t}$ over the entire horizon $[0, T]$ and not only $\left[0, t_{1}\right]$ ). Hence to improve the bounds we aim at considering choices for all $0 \leq t \leq T$. In order to do so successfully we need to impose the following condition on the market. 
Condition 1 For all $0 \leq t \leq T$ and all $i=1, \ldots, j(t)-1$ the random variables $E\left[S_{t_{i}} \mid S_{t}\right]$ are non-decreasing in $S_{t}$.

Assume that the stock price $S_{t}$ at time $t>0$ is known. Then the condition states that the expected stock prices at any intermediate time is increasing in $S_{t}$, which appears a natural and desirable property for the risky asset price process $\left\{S_{t}, t \geq 0\right\}$. From Condition 1 it follows that $A_{T}$ as defined in (5) is increasing in $S_{t}(0 \leq t \leq T)$ so that inequality (2) can be applied to find the stop-loss bound

$$
E\left[A_{T}\right]+\frac{1}{n} \sum_{i=j(t)}^{n} S_{t} e^{r\left(t_{i}-t\right)} \leq_{s l} H_{T} .
$$

where $E\left[A_{T}\right]$ is explicitly given as

$$
E\left[A_{T}\right]=\frac{1}{n} \sum_{i=1}^{j(t)-1} S_{0} e^{r t_{i}}
$$

From (7) it follows that

$$
\begin{aligned}
e^{-r T} \mathrm{E} & {\left[\operatorname{Max}\left(\mathrm{E}\left[A_{T}\right]+\frac{1}{n} \sum_{i=j(t)}^{n} S_{t} e^{r\left(t_{i}-t\right)}-K, 0\right)\right] } \\
& =e^{-r T} \frac{\sum_{i=j(t)}^{n} e^{r\left(t_{i}-t\right)}}{n} \mathrm{E}\left[\operatorname{Max}\left(S_{t}-\frac{n\left(K-\mathrm{E}\left[A_{T}\right]\right)}{\sum_{i=j(t)}^{n} e^{r\left(t_{i}-t\right)}}, 0\right)\right] \\
& =\frac{1}{n} \sum_{i=j(t)}^{n} e^{-r\left(T-t_{i}\right)} C\left(c_{t}^{(1)}, t\right) \\
& \leq A C(K, n)
\end{aligned}
$$

where $c_{t}^{(1)}$ is given as

$$
c_{t}^{(1)}=\frac{\left(n K-\sum_{i=1}^{j(t)-1} S_{0} e^{r t_{i}}\right)}{\sum_{i=j(t)}^{n} e^{r\left(t_{i}-t\right)}} .
$$

Since this holds for all $0 \leq t \leq T$ we find that

$$
L B_{t}^{(1)} \leq A C(K, n),
$$

with $L B_{t}^{(1)}$ given as

$$
L B_{t}^{(1)}=\frac{e^{-r T}}{n} \operatorname{Max}_{0 \leq t \leq T}\left(C\left(c_{t}^{(1)}, t\right) \sum_{i=j(t)}^{n} e^{r t_{i}}\right) .
$$


Note that the expression (8) can also be found in Albrecher et al (p.129 eq. (10), 2008), where more involved probabilistic arguments have been used to obtain it. Hence the value for the $L B_{t}^{(1)}$ can be observed in the market as soon as the European calls are quoted. Furthermore, in order to derive the $L B_{t}^{(1)}$ we do not really need Condition 1 . It is sufficient that (the less intuitive) condition (7) is fulfilled (see also Albrecher et al. (2008)). Finally, let us remark that $L B_{1} \leq L B_{t}^{(1)}$.

\subsubsection{Third lower bound}

The following condition usually allows to further improve the bounds.

Condition 2 For all $i=1, \ldots, j(t)-1$ we assume that $E\left[S_{t_{i}} \mid S_{t}\right] \geq$ $S_{0}^{1-\frac{t_{i}}{t}} S_{t}^{\frac{t_{i}}{t}}$ (almost surely).

Assume that the stock price $S_{t}$ at time $t>0$ is known. Then the expected stock price at intermediate times $0 \leq t_{i} \leq t$ is bounded by below by the geometric average of the stock prices $S_{t}$ and $S_{0}$ with weights equal to normalized distance between $t_{i}$ and 0 respectively. It is not difficult to show that this assumption holds when log-returns are identically and independently distributed, a condition that holds true for exponential Lévy models (see also Proposition 2.1 in Albrecher et al. (2008)).

Condition 2 implies that

$$
L_{t}:=\frac{1}{n} \sum_{i=1}^{j(t)-1} S_{0}\left(\frac{S_{t}}{S_{0}}\right)^{\frac{t_{i}}{t}}+\frac{1}{n} \sum_{i=j(t)}^{n} S_{t} e^{r\left(t_{i}-t\right)} \leq_{s l} \frac{1}{n} \sum_{i=1}^{n} S_{t_{i}}
$$

Taking into account that the left-hand side of the inequality is increasing in $S_{t}$ we find that

$$
\begin{aligned}
e^{-r T} \mathrm{E}\left[\operatorname{Max}\left(L_{t}-K, 0\right)\right] & \frac{e^{-r T} S_{0}}{n} \sum_{i=1}^{j(t)-1} \mathrm{E}\left[\operatorname{Max}\left(\left(\frac{S_{t}}{S_{0}}\right)^{\frac{t_{i}}{t}}-\left(\frac{c_{t}^{(2)}}{S_{0}}\right)^{\frac{t_{i}}{t}}, 0\right)\right] \\
& +\frac{e^{-r T}}{n} \sum_{i=j(t)}^{n} e^{r\left(t_{i}-t\right)} \mathrm{E}\left[\operatorname{Max}\left(S_{t}-c_{t}^{(2)}, 0\right)\right] \\
= & \frac{e^{-r T} S_{0}}{n} \sum_{i=1}^{j(t)-1} \mathrm{E}\left[\operatorname{Max}\left(\left(\frac{S_{t}}{S_{0}}\right)^{\frac{t_{i}}{t}}-\left(\frac{c_{t}^{(2)}}{S_{0}}\right)^{\frac{t_{i}}{t}}, 0\right)\right] \\
& +\frac{e^{-r T}}{n} \sum_{i=j(t)}^{n} e^{r t_{i}} C\left(c_{t}^{(2)}, t\right)
\end{aligned}
$$


where $c_{t}^{(2)}$ is now the solution of

$$
n K-\sum_{i=1}^{j(t)-1} S_{0}\left(\frac{c_{t}^{(2)}}{S_{0}}\right)^{\frac{t_{i}}{t}}-c_{t}^{(2)} \sum_{i=j(t)}^{n} S_{t} e^{r\left(t_{i}-t\right)}=0 .
$$

Hence we find that

$$
L B_{t}^{(2)} \leq A C(K, n)
$$

where $L B_{t}^{(2)}$ is given by

$L B_{t}^{(2)}=\frac{e^{-r T}}{n} \operatorname{Max}_{0 \leq t \leq T}\left(S_{0} \sum_{i=1}^{j(t)-1} E\left[\operatorname{Max}\left(\left(\frac{S_{t}}{S_{0}}\right)^{\frac{t_{i}}{t}}-\left(\frac{c_{t}^{(2)}}{S_{0}}\right)^{\frac{t_{i}}{t}}, 0\right)\right]+\sum_{i=j(t)}^{n} e^{r t_{i}} C\left(c_{t}^{(2)}, t\right)\right)$.

Note that in order to derive $L B_{t}^{(2)}$ we do not really need Condition 2 . It is sufficient that (the less intuitive) condition (9) will hold. Also this expression can be found in Albrecher et al. (2008) (p.132 eq. (15), 2008), but our approach is based on simpler considerations. Also note that $L B_{t}^{(2)}$ is the maximum over different time instances of prices of a path-independent power call option. Carr and Chou (1997) explain that if an investor can trade in all European calls maturing at $T$ the path-independent option can be uniquely decomposed into a bond and a continuum of calls. Hence when all call prices are available in the market the value for $L B_{t}^{(2)}$ can be determined using their decomposition formula.

In Albrecher et al. (2008) the different lower bounds for Asian option prices have been numerically evaluated with varying success. Intuitively this is because the bounds used are essentially based on "approximating" the sum $\frac{1}{n} \sum_{i=1}^{n} S_{t_{i}}$ by a conditioned sum involving a single component $S_{t_{j}}(j=$ $1,2, \ldots, n)$ only. While this procedure allows to obtain (almost) model-free lower bounds for Asian option prices, the reduction of dimensionality comes at some cost.

In this paper we aim at improving the accuracy. More precisely, we show the bounds can be used as control variates to improve efficiency of MonteCarlo approaches when estimating the price of Asian options. In doing so we focus on the third stop-loss bound. While there is no formal guarantee that it outperforms the second lower bound (and thus also the first lower bound), Albrecher et al. (2008) provides theoretical and empirical evidence that this is often the case. Recall also that this third lower bound exists in a Lévy setting and thus has practical ${ }^{4}$ appeal (see also Albrecher et al. (2008), proposition 2.1).

\footnotetext{
${ }^{4}$ It is wel-known that Lévy processes are suitable candidates to model log-returns of risky assets; see e.g. Schoutens (2003).
} 
In the next Sections 3, 4 and 5 we explain in detail the control variate approach we propose. Numerical results can be found in Section 7 .

\section{Bounds as control variates for improved simula- tion}

In this section we show that the stop-loss bounds derived in Section 2 appear as a useful device to improve Monte-Carlo simulation significantly, namely when using them as control variates.

\subsection{Control Variates}

Clearly, the pricing of an Asian option amounts to determining the expected value of a random variable. A traditional technique to cope with such problem is Monte-Carlo simulation which builds upon the law of large numbers and thereby upon large samples of random numbers. Hence, let $Y_{1}, Y_{2}, \ldots, Y_{k}$ be a series of $k$ independently and identically distributed (i.i.d.) replications of the payoff $e^{-r T}\left(\frac{1}{n} \sum_{i=1}^{n} S_{t_{i}}-K\right)^{+}$. Then, a classical estimator for $E[Y]:=e^{-r T} E\left[\left(\frac{1}{n} \sum_{i=1}^{n} S_{t_{i}}-K\right)^{+}\right]$is its sample mean $\bar{Y}$ given as

$$
\bar{Y}=\frac{1}{k} \sum_{i=1}^{k} Y_{i} .
$$

The law of large numbers assures that a sample of independent, identically distributed (i.i.d.) random variables converges to the sample mean as the sample size $k$ increases. More specifically the statistic $\bar{Y}$ is an unbiased and (strongly) consistent estimator for $E[Y]$ and,

$$
\frac{\sqrt{k}(\bar{Y}-E[Y])}{\sqrt{\operatorname{Var}[Y]}} \Rightarrow N(0,1)
$$

where " $\Rightarrow$ " denotes convergence in distribution and $N(0,1)$ denotes the standard normal distribution. To summarize: Monte-Carlo simulation consists in generating random draws $Y_{1}, \ldots, Y_{k}$ and next applying (11). We remark that the $O\left(k^{-\frac{1}{2}}\right)$ convergence rate is independent of the number of dimensions $n$ involved in the payoff sum, namely $\sum_{i=1}^{n} S_{t_{i}}$, making simulation for highly multi-dimensional problems often the preferred approach above other numerical techniques.

On the other hand, one of the main weaknesses with the Monte-Carlo method is efficiency since standard simulation provides convergence rates of (only) $k^{-1 / 2}$. Using simulation, excessive run lengths or replications may 
thus be necessary to yield estimators with acceptable precision. In practice, simulation time can be of great importance and consequently techniques have been developed to improve the performance of the Monte-Carlo method, most of which are aimed at reducing the variance of the estimator. Variance reduction techniques thus allow to obtain greater precision for the same amount of simulation time, or to achieve a desired precision with less simulation time. One of the most effective variance-reduction techniques is the method of control variates. The main idea is to use a highly correlated random variable with known mean to reduce the variance of the estimator. In our context, the geometric counterpart of the arithmetic Asian option has already been proposed in literature as a control variate by e.g. Kemna and Vorst (1990) and Fu et al. (1999). Recently, this idea was further exploited and deepened by Dingec and Hormann (2012) and Fusai and Meucci (2008).

In this paper, we show how the stop-loss bounds $L_{t}$ that we discussed in Section 2 can appear as suitable control variates. More precisely, when Condition 2 is fulfilled the random variables $L_{t}$ (see (9)) provide stop-loss bounds for $H_{T}=\frac{1}{n} \sum_{i=1}^{n} S_{t_{i}}$. We remark that the $L_{t}$ are essentially obtained through conditioning and thus may preserve a significant amount of the information contained in $H_{T}$. In other words one may intuitively expect the $L_{t}$ to have good correlation with $H_{T}$ making them possible candidates to improve convergence considerably.

First, let us consider only one time instance $t_{j}$ and therefore only one random variable $L_{t_{j}}$. Suppose that for each replication $Y_{i}$ for the payoff $e^{-r T}\left(\frac{1}{n} \sum_{i=k}^{n} S_{t_{k}}-K\right)^{+}$, we calculate another output $X_{i}$ corresponding to the replication of $X:=e^{-r T}\left(L_{t_{j}}-K\right)^{+}$(The $X_{i}$ will be later in the text denoted by $X_{i}^{\left(t_{j}\right)}$ but as long as there is no confusion just by $X_{i}$ ). Let assume that $E[X]$ is known analytically (an analytical expression is given in Section 5 in the setting of a Variance Gamma and Normal Inverse Gaussian model). We then consider the replications $X_{i}(i=1,2, \ldots, k)$ such that the subsequent pairs $\left(X_{i}, Y_{i}\right)$ are i.i.d. The unbiased estimator of $E[X]$ is therefore given by

$$
\bar{X}=\frac{1}{k} \sum_{i=1}^{k} X_{i} .
$$

Then, the control variate estimator $\overline{Y^{c}}$ of $E[Y]$, given by

$$
\begin{aligned}
\overline{Y^{c}} & =\bar{Y}-b(\bar{X}-E[X]) \\
& =\frac{1}{k} \sum_{i=1}^{k}\left(Y_{i}-b\left(X_{i}-E[X]\right)\right)
\end{aligned}
$$


is also unbiased and asymptotically normally distributed (see Glasserman (2003)).

The optimal coefficient $b^{*}$ which minimizes the variance of the new control variate estimator, namely ${ }^{5}$

$$
\operatorname{Var}\left[\overline{Y^{c}}\right]=\operatorname{Var}[\bar{Y}]\left(1-\rho_{X Y}^{2}\right)
$$

with $\rho_{X Y}$ the correlation between $X$ and $Y$, is given by (see Glasserman $(2003))$

$$
b^{*}=\frac{\operatorname{Cov}[X, Y]}{\operatorname{Var}[X]},
$$

The quantity $\operatorname{Cov}[X, Y]$ and $\operatorname{Var}[X]$ are usually not known and in that case, one has to use an estimate of $b^{*}$. Here we have chosen to use the estimate $\hat{b}$ given by

$$
\hat{b}=\frac{\sum_{i=1}^{k}\left(X_{i}-\bar{X}\right)\left(Y_{i}-\bar{Y}\right)}{\sum_{i=1}^{k}\left(X_{i}-\bar{X}\right)^{2}} .
$$

In our setting we can choose any $t_{j}$ to create a single control variate. It appears natural to use the different $L_{t_{j}}(j=1,2, \ldots, n)$ together to create $n$ control variates. Suppose that each replication of a simulation produces outputs $Y_{i}$ and $\mathbf{X}_{\mathbf{i}}=\left(X_{i}^{\left(t_{1}\right)}, \ldots, X_{i}^{\left(t_{n}\right)}\right)^{\prime}$ (the exponent ' denotes that the vector is transposed) corresponding to a vector of payoff replications, namely $\mathbf{X}=e^{-r T}\left(\left(L_{t_{1}}-K\right)^{+}, \ldots,\left(L_{t_{n}}-K\right)^{+}\right)^{\prime}$ and suppose that the vector of corresponding expectations $E[\mathbf{X}]$ is known. Then one can derive that the control variate estimator $\overline{Y^{c}}$ based on the vector of sample means of the control variates $\overline{\mathbf{X}}:=\left(\bar{X}^{\left(t_{1}\right)}, \bar{X}^{\left(t_{2}\right)}, \ldots, \bar{X}^{\left(t_{n}\right)}\right)^{\prime}$ is given by

$$
\overline{Y^{c}}=\bar{Y}-(B)^{\prime}(\overline{\mathbf{X}}-E[\mathbf{X}]),
$$

where $B^{*}=\Sigma_{\mathbf{X}}^{-1} \Sigma_{\mathbf{X} Y}$ is optimal with minimized variance of $\overline{Y^{c}} \cdot \Sigma_{\mathbf{X}}$ is a $n \mathrm{x} n$ covariance matrix and $\Sigma_{\mathbf{X} Y}$ is a $n \times 1$ covariance vector (see Glasserman (2003)). Since $B^{*}$ is not known explicitly, it needs to be estimated. In order to avoid introducing bias we first generate pairs to estimate $B^{*}$ and in particular estimates $\widehat{\Sigma}_{\mathbf{X}}$ and $\widehat{\Sigma}_{\mathbf{X} Y}$ for respectively $\Sigma_{\mathbf{X}}$ and $\Sigma_{\mathbf{X} Y}$. Next we use the remaining pairs to determine $\overline{Y^{c}}\left(B^{*}\right)$ independently, and then the minimized variance is

$$
\operatorname{Var}\left[\overline{Y^{c}}\right]=\operatorname{Var}[\bar{Y}]\left(1-R^{2}\right),
$$

where one estimates $R^{2}$ by $\widehat{\Sigma}_{\mathbf{X} Y}^{\prime} \widehat{\Sigma}_{\mathbf{X}}^{-1} \widehat{\Sigma}_{\mathbf{X} Y} / \operatorname{Var}[\bar{Y}]$.

\footnotetext{
${ }^{5}$ We denote by $(X, Y)$ a generic pair of random variables with the same distribution as each $\left(X_{i}, Y_{i}\right)$.
} 
Whereas the random variables $L_{t_{j}}$ are based on arithmetic averaging, we will propose in Subsection 3.3 control variates based on geometric averaging. These will be applied in the same way as above.

While the technique of using control variates is rather straightforward, its successful application depends on a series of conditions that need to be met. Firstly, using a control variate $X$ for estimating $E[Y]$ more efficiently implies that $E[X]$ needs to be known. Secondly, as expressed by (12) and (13) the gain in efficiency depends on the strength of correlation. Finally, on a more practical note, it is important to observe that the computational cost to generate the replications $\left(X_{i}, Y_{i}\right)$ should roughly be the same as generating the $Y_{i}$ alone. We now discuss these conditions in some more detail for the specific context at hand.

\subsection{Arithmetic Lower Bounds}

We now show that the stop-loss bounds $L_{t_{j}}$ given in (9) are usually useful as control variates in simulation schemes for pricing Asian options.

First, the intrinsic properties of the sum $L_{t_{j}}$ make it often possible to compute the $E\left[\left(L_{t_{j}}-K\right)^{+}\right](j=1, \ldots, n)$ explicitly. Indeed, as shown in Section 2 and in Albrecher et al. (2008), every $E\left[\left(L_{t}-K\right)^{+}\right]$can be expressed as

$$
E\left[\left(L_{t}-K\right)^{+}\right]=\sum_{i=1}^{j(t)-1} \frac{S_{0}^{1-\frac{t_{i}}{t}}}{n} E\left[\left(S_{t}^{\frac{t_{i}}{t}}-k^{\frac{t_{i}}{t}}\right)^{+}\right]+\sum_{i=j(t)}^{n} \frac{e^{r\left(t_{i}-t\right)}}{n} E\left[\left(S_{t}-k\right)^{+}\right]
$$

where $k$ is the solution of the equation:

$$
\frac{1}{n} \sum_{i=1}^{j(t)-1} S_{0}\left(\frac{k}{S_{0}}\right)^{\frac{t_{i}}{t}}+\frac{1}{n} \sum_{i=j(t)}^{n} k e^{r\left(t_{i}-t\right)}=K .
$$

As can be easily noticed from (14), evaluating $E\left[\left(L_{t_{j}}-K\right)^{+}\right]$essentially amounts to pricing a series of European power call payoffs of the type

$$
\left(S_{t}^{r}-K\right)^{+},
$$

with $r$ a suitable real number and $K$ a strike. We will see in Section 4 and 5 that this is often in reach explicitly.

Second, the replications for $H_{T}$ and $\mathbf{L}:=\left(L_{t_{1}}, L_{t_{2}}, \ldots, L_{t_{n}}\right)$ are both fully determined by replications for the $S_{t_{i}}(i=1,2, \ldots, n)$ so that "almost no extra cost" is implied when generating the replications $\left(H_{T}^{i}, \mathbf{L}^{i}\right)$. Third, the random variable $H_{T}$ is expected to be strongly related with the different $L_{t_{j}}(j=1,2, \ldots, n)$ so that significant efficiency gains are made. 
We remark that (9) is in fact the arithmetic average of the vector

$$
\left(S_{0}\left(\frac{S_{t}}{S_{0}}\right)^{\frac{t_{1}}{t}}, \ldots, S_{0}\left(\frac{S_{t}}{S_{0}}\right)^{\frac{t_{j(t)-1}}{t}}, S_{t} e^{r\left(t_{j(t)}-t\right)}, \ldots, S_{t} e^{r\left(t_{n}-t\right)}\right) .
$$

When using the $L_{t_{j}}(j=1,2, \ldots, n)$ as control variates to speed up the Crude Monte-Carlo (CMC) we label this in the following as the Arithmetic Lower Bound approach, and we use the shorthand notation $A L B_{t}$ to reflect $e^{-r T} E\left[\left(L_{t}-K\right)^{+}\right]$.

\subsection{Geometric Lower Bounds}

Using the lower bounds (10) at different spot times $t_{1}, \ldots, t_{n}$ to construct a multi-dimensional vector of control variates, requires the evaluation of (14) for all these spot times. This needs some computational efforts which may slow down the pricing procedures. In order to derive in a more efficient way some control variates that are both highly correlated and fast to compute, we also propose to consider the geometric average of

$$
\left(S_{0}\left(\frac{S_{t}}{S_{0}}\right)^{\frac{t_{1}}{t}}, \ldots, S_{0}\left(\frac{S_{t}}{S_{0}}\right)^{\frac{t_{j(t)-1}}{t}}, S_{t} e^{r\left(t_{j(t)}-t\right)}, \ldots, S_{t} e^{r\left(t_{n}-t\right)}\right),
$$

denoted by $\tilde{L}_{t}$, i.e.

$$
\begin{aligned}
\tilde{L}_{t}:= & \left(\prod_{i=1}^{j(t)-1} S_{0}\left(\frac{S_{t}}{S_{0}}\right)^{\frac{t_{i}}{t}} \prod_{i=j(t)}^{n} S_{t} e^{r\left(t_{i}-t\right)}\right)^{1 / n} \\
= & \alpha_{t}\left(S_{t}\right)^{\beta_{t}}
\end{aligned}
$$

with

$$
\begin{aligned}
& \alpha_{t}=\left(\prod_{i=1}^{j(t)-1}\left(S_{0}\right)^{1-\frac{t_{i}}{t}} \prod_{i=j(t)}^{n} e^{r\left(t_{i}-t\right)}\right)^{1 / n} \\
& \beta_{t}=\frac{1}{n}\left(\sum_{i=1}^{j(t)-1} \frac{t_{i}}{t}+n-j(t)+1\right) .
\end{aligned}
$$

When using the different $\tilde{L}_{t_{j}}(j=1,2, \ldots, n)$ to enhance simulation we refer to the the Geometric Lower Bound and we denote $G L B_{t}:=e^{-r T} E\left[\left(\tilde{L}_{t}-\right.\right.$ $K)^{+}$. Note that

$$
G L B_{t}:=e^{-r T} E\left[\left(\alpha_{t}\left(S_{t}\right)^{\beta_{t}}-K\right)^{+}\right] .
$$

Compared with the arithmetic lower bounds in (14), we now only need to price one European power call option for each spot time in (15). This saves a lot of computational efforts but using the geometric average is at the cost of 
the precision. Indeed, it is well known that the geometric averages are always smaller than the arithmetic ones in (almost surely) order, and therefore one also has

$$
e^{-r T} E\left[\left(\tilde{L}_{t}-K\right)^{+}\right] \leq e^{-r T} E\left[\left(L_{t}-K\right)^{+}\right] .
$$

We now proceed by specifying an important class of market models for which our pricing method based on control variates appears useful.

\section{Market setting: subordinated Brownian motion}

In this section we discuss subordinated Brownian motion as a suitable way to model asset returns. This provides a convenient framework for using the control variate approach. We will also use this setting in Section 6 where we develop our second method to price Asian options.

\subsection{Subordination}

It is convenient to express the risky asset price in terms of its logreturns, i.e. we write $S_{t}=S_{0} e^{X_{t}}$ where $X_{t}$ is to be interpreted as the cumulative logreturn. The workhorse for modelling returns consists in assuming $\left\{X_{t}\right.$, $t \geq 0\}$ is a Brownian motion with drift. This essentially amounts to assuming that the distribution of the increments $X_{t+s}-X_{s}$ over the time interval $[s, s+t], s, t \geq 0$, is normally distributed. This set-up can be traced back to Bachelier (1900) and is known in the literature as the famous Black-Scholes market. Numerous empirical studies have revealed the fact that asset price volatility tends to be time-varying and exhibits clustering effects. This in turn also implies that the assumption of normally distributed returns is not suitable to capture the long-tailed features of financial time series, especially when the problem at hand involves short-term returns; see e.g. Mandelbrot (1963), Eberlein et al. (1998) or Carr et al. (2002).

An idea to accommodate for this is to generalize the Brownian motion by making time itself stochastic, i.e. by considering time-changed Brownian motions. To this end suppose that $\phi(u)$ is the characteristic function related to some distribution function (d.f.) and let $Z_{t}=\theta G_{t}+\sigma B_{G_{t}}$ where $\theta \in \mathbb{R}, \sigma \in \mathbb{R}_{0}^{+}, B_{t}$ is the standard Brownian motion and where $\left\{G_{t}, t \geq 0\right\}$ is another positive stochastic process. The latter process is referred to as time-change, stochastic clock, chronometer or business time. We assume it starts at zero, has independent and stationary increments and is such that the distribution of an increment over $[s, s+t], s, t \geq 0$, i.e. $G_{t+s}-G_{s}$, has $(\phi(u))^{t}$ as its characteristic function. If $\left\{G_{t}, t \geq 0\right\}$ is a positive and strictly 
increasing Lévy process it is called a subordinator. It can be shown that a Lévy process that is time-changed by a subordinator remains a Lévy process (see Sato (1999)).

The process $\left\{Z_{t}, t \geq 0\right\}$ constructed in the way above from a Brownian motion is called a subordinated Brownian motion. Such processes provide more flexibility and potential accuracy when modelling stochastic returns. For a full theoretical background we refer to Cont and Tankov (2004). The focus on subordinated Brownian motions for modelling asset returns is no real restriction because Monroe (1978) essentially showed that arbitragefree models for assets can be represented as time-changed Brownian motions. Note that many popular models in finance are based on time-changed Brownian motion where the time-change is chosen to be a subordinator. E.g. the Variance Gamma process or the Generalized Hyperbolic Model (including the Normal Inverse Gaussian process) can be represented as Brownian motion time-changed by resp. a Gamma process (Madan et al. (1998)) or a Generalized Inverse Gaussian process (Eberlein and Keller (1995)). The Normal Tempered Stable process (also including the Normal Inverse Gaussian process) can be written as Brownian motion time-changed by a tempered stable subordinator. We now formally present the first two models mentioned.

\subsection{The Variance Gamma model}

This process was originally introduced by Madan and Senata (1990) and further studied in Madan et al. (1998). It is a pure jump process that is obtained by changing the clock of a standard Brownian motion by a Gamma process. More precisely, let $\left\{B_{t}, t \geq 0\right\}$ denote a standard Brownian motion, $\sigma>0$ and $\theta \in \mathbb{R}$; then the Variance Gamma process $Z^{V G}=\left\{Z_{t}^{V G}, t \geq 0\right\}$, with parameters $\sigma, \nu$ and $\theta$ is defined as

$$
Z_{t}^{V G}=\theta G_{t}+\sigma B_{G_{t}}
$$

where $G_{t}$ is a Gamma process with mean $t$ and variance $\nu t$. The density of $G_{t}$ can be written as

$$
f_{G t}\left(x ; \frac{t}{\nu}, \nu\right)=x^{\frac{t}{\nu}-1} \frac{e^{-\frac{x}{\nu}}}{\Gamma\left(\frac{t}{\nu}\right) \nu^{\frac{t}{\nu}}}, \quad \nu>0,
$$

where $\Gamma(\cdot)$ stands for the Gamma function.

The density and the characteristic function of a Brownian motion timechanged by a subordinator, $G_{t}$, can be derived exploiting the fact that $Z_{t}$ conditional on $G_{t}=g$ has a Gaussian distribution with mean $\theta g$ and variance 
$\sigma^{2} g$. For completeness we report the characteristic and the density function in case of univariate Variance Gamma:

$$
\begin{aligned}
\varphi_{V G}(u ; \sigma, \nu, \theta) & =\left(1-i u \nu \theta+\frac{1}{2} \nu u^{2} \sigma^{2}\right)^{-\frac{t}{\nu}} \\
f_{V G}(x ; \sigma, \nu, \theta) & \left.=\frac{2 e^{\frac{\theta x}{\sigma^{2}}}}{\nu^{\frac{t}{\nu}} \sqrt{2 \pi} \sigma \Gamma\left(\frac{t}{\nu}\right)}\left(\frac{x^{2}}{\frac{2 \sigma^{2}}{\nu}+\theta^{2}}\right)^{\frac{t}{2 \nu}-\frac{1}{4}} K_{\frac{t}{\nu}-\frac{1}{2}}\left(\frac{1}{\sigma^{2}} \sqrt{x^{2}\left(\frac{2 \sigma^{2}}{\nu}+\theta^{2}\right.}\right)\right)
\end{aligned}
$$

where $K_{k}(\cdot)$ denotes the modified Bessel function of the second kind of order $k$, see e.g. Madan et al. (1998).

In a Lévy market, there are many different equivalent martingale measures to choose. There exist two popular approaches to find an equivalent martingale measure, namely by using the so-called Esscher transform (see Gerber and Shiu (1994) and the next paragraph) or by mean-correcting the exponential of a Lévy process (see Schoutens (2003)). This last method consists in changing the "drift" parameter in the Lévy process such that the discounted stock-price process becomes a martingale. For the Variance Gamma model, we follow this last approach as in the paper of Albrecher et al. (2008) since we aim to use their parameter estimates in our numerical section.

More precisely, the risk neutral process for the stock price dynamics in this section is given by the following VG process which is obtained by replacing the role of the Brownian motion in the original Black-Scholes model by the VG process and by taking into account the right drift such that the discounted stock-price process is a martingale:

$$
\begin{aligned}
S_{t} & =S_{0} \exp \left((r+\omega) t+Z_{t}^{V G}\right) \\
& =S_{0} \exp \left(\widetilde{Z}_{t}^{V G}\right)
\end{aligned}
$$

where $\widetilde{Z}_{t}^{V G}=(r+\omega) t+Z_{t}^{V G}$ with $\omega=\frac{1}{\nu} \ln \left(1-\theta \nu-\frac{\sigma^{2} \nu}{2}\right)$. Indeed, in this case one easily observes that the mean rate of return on the stock equals the risk-free interest rate $r$.

\subsection{The Normal Inverse Gaussian model}

The Normal Inverse Gaussian (NIG) process $Z^{N I G}=\left\{Z_{t}^{N I G}, t \geq 0\right\}$ has stationary and independent NIG distributed increments with $Z_{0}^{N I G}=0$. More precisely, $Z_{t}^{N I G}$ has a NIG law with parameters $\alpha, \eta, \delta$ and $\mu$ given by 


$$
f_{\mathrm{NIG}}(x ; \alpha, \eta, \delta, \mu)=\frac{\alpha \delta}{\pi} e^{\delta \sqrt{\alpha^{2}-\eta^{2}}+\eta(x-\mu)} \frac{K_{1}\left(\alpha \sqrt{\delta^{2}+(x-\mu)^{2}}\right)}{\sqrt{\delta^{2}+(x-\mu)^{2}}}
$$

with $0 \leq|\eta| \leq \alpha, \delta \geq 0, \mu \in \mathbb{R}$, where $K_{1}(\cdot)$ denotes the modified Bessel function of the second kind of order 1 . For a survey on stochastic processes of normal inverse Gaussian type, we refer to Barndorff-Nielsen (1998).

It is further well known (see e.g. Schoutens (2003)) that a NIG process $Z_{t}^{N I G}$ with parameters $\alpha, \eta, \delta$ and $\mu$ with density (20) can be rewritten as a drift term plus an Inverse Gaussian time-changed Brownian motion, namely as

$$
Z_{t}^{N I G}=\mu t+\eta \delta^{2} I_{t}+\delta B_{I_{t}}
$$

where $\left\{I_{t}, t \geq 0\right\}$ is an Inverse Gaussian process for which the density function is explicitly known, namely

$$
f_{I G}(x ; a, b)=\frac{a}{\sqrt{2 \pi}} e^{a b} x^{-3 / 2} e^{-\frac{1}{2}\left(a^{2} x^{-1}+b^{2} x\right)}, x>0
$$

with parameters $a=1$ and $b=\delta \sqrt{\alpha^{2}-\eta^{2}}$.

To apply the Normal Inverse Gaussian model (NIG) for market price modelling, we recall from Albrecher and Predota (2004) that log-returns of asset prices will be modeled by a NIG process $Z_{t}^{N I G}$, and therefore prices by $S_{t}=S_{0} \exp \left(Z_{t}^{N I G}\right)$. Since the market model is incomplete, there are many candidates of equivalent martingale measures for risk neutral valuation and we use as in the paper of Albrecher and Predota (2004) the method of Esscher transforms in order to be able to use their parameter estimates later on. We refer to Albrecher and Predota (2004) for details and further references.

In particular Albrecher and Predota (2004) shows that for a Lévy process $\left\{Z_{t}^{N I G}, t \geq 0\right\}$ it is possible to define a locally equivalent probability measure $P^{\theta}$, called the Esscher equivalent measure, through

$$
d P^{\theta}=\exp \left(\theta Z_{t}^{N I G}-t \log M_{N I G}(\theta)\right) d P,
$$

with $M_{N I G}(\theta)$ the moment generating function of $(20)^{6}$. This probability measure $P^{\theta}$ is a risk neutral probability measure if $\theta$ is the solution of

$$
r=\mu+\delta\left(\sqrt{\alpha^{2}-(\eta+\theta)^{2}}-\sqrt{\alpha^{2}-(\eta+\theta+1)^{2}}\right) .
$$

\footnotetext{
${ }^{6}$ As mentioned in footnote 2 , we assume tacitly the existence of all expectations, and moreover of all moment generating functions.
} 
The stock prices under this probability measure $P^{\theta}$ also follow a NIG process with new parameter $\eta^{\prime}=\eta+\theta$. Therefore, under this risk neutral measure, we consider $S_{t}=S_{0} \exp \left(\widetilde{Z}_{t}^{N I G}\right)$ where $\widetilde{Z}_{t}^{N I G}$ is a $\operatorname{NIG}(\alpha, \eta+\theta, \delta, \mu)$ process.

Note that the NIG distribution is a special case $\lambda=-\frac{1}{2}$ of the generalized hyperbolic distribution given by the density

$$
\begin{aligned}
f_{\mathrm{GH}}(x ; \alpha, \eta, \delta, \lambda, \mu) & =a(\alpha, \eta, \delta, \lambda) \frac{K_{\lambda-\frac{1}{2}}\left(\alpha \sqrt{\delta^{2}+(x-\mu)^{2}}\right)}{\left(\delta^{2}+(x-\mu)^{2}\right)^{\frac{1}{4}-\frac{\lambda}{2}}} e^{\eta(x-\mu)}, \\
a(\alpha, \eta, \delta, \lambda) & =\frac{\left(\alpha^{2}-\eta^{2}\right)^{\frac{\lambda}{2}}}{\sqrt{2 \pi} \alpha^{\lambda-\frac{1}{2}} \delta^{\lambda} K_{\lambda}\left(\delta \sqrt{\alpha^{2}-\eta^{2}}\right)}
\end{aligned}
$$

where $K_{\nu}(x)$ denotes as above the modified Bessel function of the second kind of order $\nu$. Derivation of the characteristic function of the GH distribution (as well as its Lévy measure) can be found in Prause (1999), namely

$$
\varphi_{\mathrm{GH}}(u ; \alpha, \eta, \delta, \lambda, \mu)=e^{i u \mu}\left(\frac{\alpha^{2}-\eta^{2}}{\alpha^{2}-(\eta+i u)^{2}}\right)^{\frac{\lambda}{2}} \frac{K_{\lambda}\left(\delta \sqrt{\alpha^{2}-(\eta+i u)^{2}}\right)}{K_{\lambda}\left(\delta \sqrt{\alpha^{2}-\eta^{2}}\right)}
$$

The generalized hyperbolic process is a pure jump process that has been introduced in finance by the work of Eberlein and Keller (1995). Its construction is similar to the one of the Variance Gamma process and the NIG process. However, this time the stochastic clock follows a generalized inverse Gaussian distribution.

The Variance Gamma process can also be seen as a special case of the generalized hyperbolic process. The density and characteristic function (18) can be obtained from the GH density and characteristic function namely (21) and (22) by taking $\alpha=\sqrt{(2 / \nu)+\left(\theta^{2} / \nu^{4}\right)}, \eta=\theta / \sigma^{2}, \lambda=\sigma^{2} / \nu, \mu=0$ and $\delta \rightarrow 0$, see e.g. Schoutens (2003).

\section{$5 \quad$ ALB and GLB as an expression of power calls}

We remark that both the ALB and GLB are based on the evaluation of power call options with price

$$
e^{-r T} E\left[\left(\alpha_{t}\left(S_{t}\right)^{\beta_{t}}-K\right)^{+}\right] .
$$


Indeed, the value of $\mathrm{GLB}_{t}$ is then immediately obtained by substituting the expressions of $\alpha_{t}$ and $\beta_{t}$ stated in (16). The value of $\mathrm{ALB}_{t}$ follows from rewriting (14) as follows

$$
\begin{array}{r}
e^{-r T} E\left[\left(L_{t}-K\right)^{+}\right]=e^{-r T} \sum_{i=1}^{j(t)-1} \frac{S_{0}^{1-\frac{t_{i}}{t}}}{n} E\left[\left(\alpha_{t_{i}}\left(S_{t}\right)^{\beta_{t_{i}}}-k^{\frac{t_{i}}{t}}\right)^{+}\right] \\
+e^{-r T} \sum_{i=j(t)}^{n} \frac{e^{r\left(t_{i}-t\right)}}{n} E\left[\left(\alpha_{t_{i}}\left(S_{t}\right)^{\beta_{t_{i}}}-k\right)^{+}\right],
\end{array}
$$

with $\alpha_{t_{i}}=1$ for all $i, \beta_{t_{i}}=\frac{t_{i}}{t}$ for all $1 \leq i \leq j(t)-1, \beta_{t_{i}}=1$ for all $j(t) \leq i$; and with the appropriate strikes (see Subsections 3.2 and 3.3).

We now will derive expressions for (23) in the Variance Gamma model and in the Normal Inverse Gaussian model.

\subsection{Calculation of power call options in the Variance Gamma model}

We recall from Section 4 that under the chosen risk neutral measure the risk neutral process is given by (19), namely

$$
S(t)=S_{0} \exp \left(\widetilde{Z}_{t}^{V G}\right)
$$

where $\widetilde{Z}_{t}^{V G}=(r+\omega) t+Z_{t}^{V G}, \omega=\frac{1}{\nu} \ln \left(1-\theta \nu-\frac{\sigma^{2} \nu}{2}\right)$, and where $\left\{Z_{t}^{V G}, t \geq 0\right\}$ is a Variance Gamma process with parameters $\sigma, \nu$ and $\theta$.

As mentioned in the beginning of this section, we study essentially the price of a general power call option, namely

$$
\begin{aligned}
e^{-r T} E\left[\left(\alpha_{t}\left(S_{t}\right)^{\beta_{t}}-K\right)^{+}\right] & =e^{-r T} E\left[\left(\alpha_{t}\left(S_{0}\right)^{\beta_{t}} e^{\left.\left.\beta_{t} \widetilde{Z}_{t}^{V G}-K\right)^{+}\right]}\right.\right. \\
& =e^{-r T} E\left[E\left[\left(\alpha_{t}\left(S_{0}\right)^{\beta_{t}} e^{\beta_{t} \widetilde{Z}_{t}^{V G}}-K\right)^{+} \mid G(t)=g\right]\right] \\
& =e^{-r T} E\left[\int_{k^{*}}^{+\infty}\left(\alpha_{t}\left(\xi_{t}\right)^{\beta_{t}} e^{\beta_{t} x}-K\right) f(x) d x\right]
\end{aligned}
$$

where $k^{*}=\frac{1}{\beta_{t}} \ln \left(\frac{K}{\alpha_{t}\left(\xi_{t}\right)^{\beta_{t}}}\right), \xi_{t}=S_{0} \exp (r t+\omega t)$ is a deterministic factor and where $f(x)$ is the Gaussian probability density function with mean $\theta g$ and variance $\sigma^{2} g$.

Hence the inner expectation is given by the following Black-Scholes type formula, denoted by $\Phi(g)$ : 


$$
\begin{aligned}
\Phi(g): & =\int_{k^{*}}^{+\infty}\left(\alpha_{t}\left(\xi_{t}\right)^{\beta_{t}} e^{\beta_{t} x}-K\right) f(x) d x \\
= & \alpha_{t}\left(\xi_{t}\right)^{\beta_{t}} \exp \left(\beta_{t} \theta g+\frac{\sigma^{2} g \beta_{t}^{2}}{2}\right)\left(1-\mathcal{N}\left(k^{*} ; \theta g+\sigma^{2} g, \sigma \sqrt{g}\right)\right) \\
& \quad-K\left(1-\mathcal{N}\left(k^{*} ; \theta g, \sigma \sqrt{g}\right)\right)
\end{aligned}
$$

where $\mathcal{N}(x ; \mu, \sigma)$ is the Gaussian cumulative distribution function with mean $\mu$ and variance $\sigma^{2}$.

With this notation, the price of a general power call option equals

$$
e^{-r T} E\left[\left(\alpha_{t}\left(S_{t}\right)^{\beta_{t}}-K\right)^{+}\right]=e^{-r T} \int_{0}^{\infty} \Phi(g) f_{G(t)}(g) d g
$$

Using the density function $f_{G(t)}(g)$ of $(17)$ and by substituting $y=\frac{g}{\nu}$, one easily finds the following expression:

$$
\begin{aligned}
e^{-r T} E\left[\left(\alpha_{t}\left(S_{t}\right)^{\beta_{t}}-K\right)^{+}\right] & =\int_{0}^{\infty} \Phi(y \nu) y^{\frac{t}{\nu}-1} \nu^{\frac{t}{\nu}-1} \frac{e^{-y}}{\Gamma\left(\frac{t}{\nu}\right) \nu^{\frac{t}{\nu}}} \nu d y \\
& =\int_{0}^{\infty} y^{\frac{t}{\nu}-1} e^{-y} \frac{\Phi(y \nu)}{\Gamma\left(\frac{t}{\nu}\right)} d y
\end{aligned}
$$

This integral can be computed numerically by using the generalized Gauss Laguerre integration method. Indeed, the generalized Gauss Laguerre quadrature (GLQ) is a numerical method to evaluate integrals of the form $\int_{0}^{+\infty} w(y ; \alpha) f(y) d y$ where $w(y ; \alpha)=y^{\alpha} e^{-y}$ with $\alpha>-1$ (so in our case $f(y)=\frac{\Phi(y \nu)}{\Gamma\left(\frac{t}{\nu}\right)}$ and $\left.\alpha=\frac{t}{\nu}-1\right)$. More precisely, the Gauss Laguerre quadrature proposes the following approximation

$$
\int_{0}^{+\infty} y^{\alpha} e^{-y} f(y) d y \approx \sum_{i}^{n} w_{i} f\left(y_{i}\right)
$$

where $y_{i}$ are the zeros of the generalized Laguerre polynomial $L_{n}^{(\alpha)}(x)$ (see Abramowitz and Stegun (1964)). These polynomials can be evaluated by using the following recurrence relation:

$$
(n+1) L_{n+1}^{(\alpha)}(y)=(2 n+1+s-y) L_{n}^{(\alpha)}(y)-(n+s) L_{n-1}^{(\alpha)}(y)
$$

with initial conditions $L_{0}^{(\alpha)}(y)=1$ and $L_{-1}^{(\alpha)}(y)=0$. The weights $\omega_{i}$ are given by the formula 


$$
w_{i}=\frac{-\Gamma(n+\alpha)}{\Gamma(n+1) L_{n-1}^{(\alpha)}\left(x_{i}\right) \frac{d L_{n}^{(\alpha)}}{d x}\left(x_{i}\right)}
$$

where the derivatives can be obtained by using the following relation:

$$
y \frac{d}{d x} L_{n}^{(\alpha)}(y)=n L_{n}^{(\alpha)}(y)-(n+s) L_{n-1}^{(\alpha)}(y) .
$$

For more details about this method, we refer to Press et al. (1992) and Stroud and Secrest (1966). There are already a few papers that successfully applied GLQ for pricing various derivatives under the VG model in an accurate and fast way (see for example Madan et al. (2011) and Garcia et al. (2007)). Browne et al. (2003) used generalized GLQ for liquidity premium calculation. In our work, as we have explained above, the generalized GLQ is a good method to compute equation (24) showing up in the ALB and GLB values. The generalized GLQ leads to accurate and fast approximations.

We remark that another famous and fast method to compute call options is the Fast Fourrier Transform (FFT), see e.g. Carr and Madan (1998). Indeed, one could use FFT to compute expressions of the form (23). However, the FFT is adequate if one wants to price options for multiple strikes because it returns a vector of option prices simultaneously for different strikes. This makes it a suitable approach in the context of model calibration for instance. But in our case we need only the price of one option for a fixed strike for different $\alpha_{t}$ and $\beta_{t}$. Hence, an integration routine which concentrates on single option prices saves significant computation time. Therefore, we prefer the generalized Gauss Laguerre quadrature.

\subsection{Calculation of power call options in the Normal Inverse Gaussian model}

To apply the Normal Inverse Gaussian model (NIG), we recall from Subsection 4.3 that we model stock prices by $S_{t}=S_{0} \exp \left(\widetilde{Z}_{t}^{N I G}\right)$, where $\widetilde{Z}_{t}^{N I G}$ is a $\operatorname{NIG}(\alpha, \eta+\theta, \delta, \mu)$ process under a well-chosen risk neutral measure, related to the Esscher transform.

Along the same lines as before we find that

$$
\begin{aligned}
& e^{-r T} E\left[\left(\alpha_{t}\left(S_{t}\right)^{\beta_{t}}-K\right)^{+}\right] \\
& =e^{-r T} \int_{\frac{1}{\beta_{t}} \ln \left(\frac{K}{\alpha_{t} S_{0}^{\beta_{t}}}\right)}^{\infty}\left(\alpha_{t} S_{0}^{\beta_{t}} e^{\beta_{t} x}-K\right) f_{\mathrm{NIG}}(x ; \alpha, \eta+\theta, \delta, \mu) d x
\end{aligned}
$$

Note that $\exp \left(\beta_{t} x\right) f_{\mathrm{NIG}}(x ; \alpha, \eta+\theta, \delta, \mu)$ can be rewritten as

$$
f_{\mathrm{NIG}}\left(x ; \alpha, \eta+\theta+\beta_{t}, \delta, \mu\right) c_{t}^{\prime},
$$


where

$$
c_{t}^{\prime}=e^{\delta_{t}\left(\sqrt{\alpha^{2}-(\eta+\theta)^{2}}-\sqrt{\alpha^{2}-\left(\eta+\theta+\beta_{t}\right)^{2}}\right)+\beta_{t} \mu .}
$$

Therefore, one easily can derive an expression based on the cumulative distribution functions $F_{\mathrm{NIG}}(\cdot)$ :

$$
\begin{aligned}
e^{-r T} E\left[\left(\alpha_{t}\left(S_{t}\right)^{\beta_{t}}-K\right)^{+}\right] & \\
= & e^{-r T} c_{t}^{\prime} \alpha_{t} S_{0}^{\beta_{t}}\left(1-F_{\mathrm{NIG}}\left(\frac{1}{\beta_{t}} \ln \left(\frac{K}{\alpha_{t} S_{0}^{\beta_{t}}}\right) ; \alpha, \eta+\theta+\beta_{t}, \delta, \mu\right)\right) \\
& \quad-K\left(1-F_{\mathrm{NIG}}\left(\frac{1}{\beta_{t}} \ln \left(\frac{K}{\alpha_{t} S_{0}^{\beta_{t}}}\right) ; \alpha, \eta+\theta, \delta, \mu\right)\right) .
\end{aligned}
$$

\section{Lower bound derived using the stochastic clock}

Inspired by the idea of conditioning used to derive the model-independent bounds in Section 2, we now present another method. Indeed, we will condition on different values in the path of the subordinator, also called the stochastic clock. Assuming that $S_{t}=S_{0} \exp \left(\mu t+\theta G_{t}+\sigma B_{G_{t}}\right)$, where $B_{t}$ is a Brownian motion and where $\left\{G_{t}, t \geq 0\right\}$ is the stochastic clock, we can write the average $\frac{1}{n} \sum_{i=1}^{n} S_{t_{i}}$ as $\sum_{i=1}^{n} \alpha\left(t_{i}, G_{t_{i}}\right) \exp \left(\sigma B_{G_{t_{i}}}\right)$, with $\alpha\left(t_{i}, G_{t_{i}}\right)=$ $\frac{S_{0} e^{\mu t_{i}+\theta G_{t_{i}}}}{n}$. As before, we assume that $t_{n}=T$.

Using this notation, we can express the price of the Asian call option at time $t=0$ as

$$
\begin{aligned}
A C(K, n) & =e^{-r T} E\left[\left(\frac{1}{n} \sum_{i=1}^{n} S_{t_{i}}-K\right)^{+}\right] \\
& =e^{-r T} E\left[E\left[\left(\sum_{i=1}^{n} \alpha\left(t_{i}, G_{t_{i}}\right) \exp \left(\sigma B_{G_{t_{i}}}\right)-K\right)^{+} \mid G_{t_{1}}, \ldots, G_{t_{n}}\right]\right] \\
& =e^{-r T} E\left[E_{\mathbf{G}}\left[\left(\sum_{i=1}^{n} \alpha\left(t_{i}, G_{t_{i}}\right) \exp \left(\sigma B_{G_{t_{i}}}\right)-K\right)^{+}\right]\right]
\end{aligned}
$$

where $E_{\mathbf{G}}[$.$] is a short-hand notation to reflect E\left[. \mid G_{t_{1}}, \ldots, G_{t_{n}}\right]$.

Note that by conditioning, the inner expectation is essentially an option on an average of lognormally distributed random variables. Indeed, after conditioning we have that $\sigma B_{G_{t_{i}}}$ are normally distributed r.v.'s with means and variances that only depend on the values of $G_{t_{i}}$. 
Vanduffel et al. (2008a) proposed for $E\left[\left(\sum_{i=1}^{n} \alpha_{i} e^{X_{i}}-K\right)^{+}\right]$with $X_{i}$ (multi-variate) normal r.v.'s, a lower bound based on the so-called maximal variance approximation, namely

$$
E\left[\left(E\left[\sum_{i=1}^{n} \alpha_{i} e^{X_{i}} \mid \Lambda=\sum_{i=1}^{n} \lambda_{i} X_{i}\right]-K\right)^{+}\right],
$$

with an appropriate choice for the coefficients of the conditioning variable $\Lambda=\sum_{i=1}^{n} \lambda_{i} X_{i}$. Indeed, the $\lambda_{i}$ are chosen in order to maximize the first order approximation of the variance of $E\left[\sum_{i=1}^{n} \alpha_{i} e^{X_{i}} \mid \Lambda=\sum_{i=1}^{n} \lambda_{i} X_{i}\right]$. This method leads to lower very accurate, analytical lower bound approximations of the true prices (see e.g. Kaas et al. (2000), Vanduffel et al. (2008a), Vanduffel et al. (2008b) and Valdez et al. (2009)).

Applying the appropriate formulae of Vanduffel et al. (2008a), namely their equations (10), (17), (37) and (40), we derive the lower bound

$$
\begin{aligned}
& A C(K, n)=e^{-r T} E\left[E_{\mathbf{G}}\left[\left(\sum_{i=1}^{n} \alpha\left(t_{i}, G_{t_{i}}\right) \exp \left(\sigma B_{G_{t_{i}}}\right)-K\right)^{+}\right]\right] \\
& \geq e^{-r T} E\left[E_{\mathbf{G}}\left[\left(E\left[\sum_{i=1}^{n} \alpha\left(t_{i}, G_{t_{i}}\right) \exp \left(\sigma B_{G_{t_{i}}}\right) \mid \Lambda=\sum_{i=1}^{n} \lambda_{i} \sigma B_{G_{t_{i}}}\right]-K\right)^{+}\right]\right] \\
& =e^{-r T} E\left[E _ { \mathbf { G } } \left[\sum _ { i = 1 } ^ { n } \alpha ( t _ { i } , G _ { t _ { i } } ) E \left[e^{\left.\left.\left.\sigma B_{G_{t_{i}}}\right] \mathcal{N}\left(R_{G_{t_{i}}}-\mathcal{N}^{-1}(p)\right)-K(1-p)\right]\right]}\right.\right.\right.
\end{aligned}
$$

with $\lambda_{i}=\alpha\left(t_{i}, G_{t_{i}}\right) E\left[e^{\sigma B_{G_{t_{i}}}}\right]=\alpha\left(t_{i}, G_{t_{i}}\right) \exp \left(E\left[\sigma B_{G_{t_{i}}}\right]+\frac{1}{2} \operatorname{Var}\left[\sigma B_{G_{t_{i}}}\right]\right)$,

$$
\begin{aligned}
R_{G_{t_{i}}} & =\frac{\sum_{j=1}^{n} \alpha\left(t_{i}, G_{t_{i}}\right) E\left[e^{\left.\sigma B_{G_{t_{i}}}\right] \operatorname{cov}\left[B_{G_{t_{i}}}, B_{G_{t_{j}}}\right] \sigma^{2}}\right.}{\sigma_{\Lambda}} \\
\sigma_{\Lambda}^{2} & =\sum_{i=1}^{n} \sum_{j=1}^{n} \alpha\left(t_{i}, G_{t_{i}}\right) \alpha\left(t_{j}, G_{t_{j}}\right) E\left[e^{\sigma B_{G_{i}}}\right] E\left[e^{\sigma B_{G_{t_{j}}}}\right] \operatorname{cov}\left[B_{G_{t_{i}}}, B_{G_{t_{j}}}\right] \sigma^{2}
\end{aligned}
$$

and with $p$ the solution of the equation

$$
Q_{p}\left[E_{\mathbf{G}}\left[\sum_{i=1}^{n} \alpha\left(t_{i}, G_{t_{i}}\right) \exp \left(\sigma B_{G_{t_{i}}}\right) \mid \Lambda=\sum_{i=1}^{n} \lambda_{i} \sigma B_{G_{t_{i}}}\right]\right]=K
$$

where $Q_{p}(X)$ is the $p$-quantile risk measure for a r.v. $X$ defined as

$$
Q_{p}(X)=\inf \left\{x \in \mathbb{R} \mid F_{X}(x) \geq p\right\}, \quad p \in(0,1),
$$

where $F_{X}(x)=P[X \leq x]$ and $\mathcal{N}(\cdot)$ the cdf of a standard normal distribution. 
Since the expressions in (25) only depend on the values $G_{t_{i}}$ of the path of the subordinator, we can denote $(25)$ as $e^{-r T} E\left[H\left(G_{t_{1}}, \ldots, G_{t_{n}}\right)\right]$ for a certain function $H$ given by

$$
H\left(G_{t_{1}}, \ldots, G_{t_{n}}\right):=E_{\mathbf{G}}\left[\sum_{i=1}^{n} \alpha\left(t_{i}, G_{t_{i}}\right) E\left[e^{\sigma B_{G_{t_{i}}}}\right] \mathcal{N}\left(R_{G_{t_{i}}}-\mathcal{N}^{-1}(p)\right)-K(1-p)\right] .
$$

Therefore, the lower bound (25) could be calculated by a numerical multidimensional integration method. However, since there is usually a large number of spot times to take into account, one would need a high-dimensional numerical integration method which usually turns out to be slow and moreover not always precise due to the accumulated errors. Therefore, in this paper, we use a Monte-Carlo simulation with GLB as control variates to compute the values in (25). This method will be referred to as the Lower Bound derived by using the Stochastic Clock (LBSC). As explained before, such a Monte-Carlo simulation is easy to implement, and as our numerical results in Section 7 will show, this method leads to very precise approximations. This method has the advantage that one has not to simulate Brownian motions. Indeed, for this approach one only needs to generate the values of the clock and of the analytical formula (26). Therefore, one only needs to generate half the numbers in comparison with the case of time-changed Brownian motion. Furthermore, the variance of the conditional expectation $H\left(G_{t_{1}}, \ldots, G_{t_{n}}\right)$ is smaller than the variance of the Asian payoff so that convergence is expected to be much faster than the crude Monte-Carlo (see also Section 7.2.3).

\section{$7 \quad$ Numerical illustrations}

In this section, we present different sets of numerical results in order to illustrate the methods introduced in this paper. We focus on the two subordinated Brownian motion Lévy models that were described in Section 4, namely the variance gamma (VG) and normal inverse Gaussian (NIG) processes. For deriving the numerical results, we made use of the CPU Intel Core i7-3630 QM 2.40GHz and RAM 8.00GB under the Windows8 OS 64 bit.

\subsection{General Set-up}

We present our numerical results for Asian options in the context of a VG economy (see Subsection 7.2.1) and a NIG economy (see Subsection 7.2.2). We use a crude Monte-Carlo (CMC) approach as the benchmark to compare the results of the Monte-Carlo approaches that use as control variates the Arithmetic Lower Bound (ALB), resp. the Geometric Lower Bound (GLB) 
as presented in Section 3, with the results of the semi-analytic lower bound approach (LBSC) that was explained in Section 6. In all instances, we use the same 50,000 simulations to perform the calculations. Specifically, for the different methods mentioned we provide the estimated mean, the standard error (S.E.), the computation time (C), the variance reduction factor (Var$\mathrm{RF})$ and the efficiency reduction factor (Eff-RF). These parameters are wellknown and do not require further explanation except the last two.

The variance reduction factor (Var-RF) is defined as the ratio between the variance of the estimator for the method at hand and the variance of the estimator under the crude Monte-Carlo approach. Note indeed that variance reduction techniques precisely allow to obtain greater precision for the same budget of computer time, or, equivalently, to obtain a given degree of precision with less simulation time. Therefore, it is natural to consider the variance reduction factor as a first performance measure. However, to measure the efficiency of approaches, we should not only consider the variance reduction but also the computation time and the possibility of bias. Let $\mu$ be the unknown quantity that we estimate (i.e. the mean of the control variate at hand) and let $\bar{Y}$ be the corresponding estimator. The bias ${ }^{7}$, variance and mean square error of $\bar{Y}$ are defined as,

$$
\begin{aligned}
\operatorname{Bias}(\bar{Y}) & =E[\bar{Y}]-\mu, \\
\operatorname{Var}(\bar{Y}) & =E\left[(\bar{Y}-E[\bar{Y}])^{2}\right], \\
\operatorname{MSE}(\bar{Y}) & =E\left[(\bar{Y}-\mu)^{2}\right]=(\operatorname{Bias}(\bar{Y}))^{2}+\operatorname{Var}(\bar{Y}),
\end{aligned}
$$

where the mean square error reflects both the bias and the variance. A second possible measure (see Glasserman (2003) and L'Ecuyer (1994)) to reflect the efficiency (Eff) of an estimator $\bar{Y}$ is then to use the inverse of the average computation time $(\mathrm{C})$ multiplied by the mean square error:

$$
\operatorname{Eff}(\bar{Y})=\frac{1}{C(\bar{Y}) \operatorname{MSE}(\bar{Y})} .
$$

This efficiency measure balances the issues of computation time, bias and variance reduction and has a strong intuitive appeal. Note however that computation time also depends on the efficiency of the particular code that is used and computer specifications. Furthermore, the penalty for bias in this

\footnotetext{
${ }^{7}$ In this paper we do not consider the bias that arises because of the error caused by the numerical method at hand, but only the bias that appears if one uses a biased estimator. In other words, we only consider the bias when using the LBSC approach to approximate the true value (see Section 6). However, note that all other control variate based methods that we discuss are also biased to some extent even when the estimator used is unbiased. Indeed, numerical integration methods such as the Gauss Laguerre quadrature or Fast Fourier Transforms also have an error term as well and are therefore biased.
} 
measure appears in an ad-hoc way and lacks a strong theoretical foundation. In some situations (e.g. in the context of risk management) a biased approach may be perfectly acceptable to some degree whereas in other cases (e.g. a trading context) this feature may be considered as completely unacceptable. In other words, the precise interpretation of the efficiency reduction factor (Eff-RF) also depends on the context at hand. This remark also explains why we in the numerical illustrations we also provide the computation time for all methods used, and also why we separately show the (estimated) relative bias for the LBSC approach. Formally, the relative bias (Rel. Bias) is defined as

$$
\text { Rel. Bias }=\left|\frac{E\left[\bar{Y}_{\mathrm{LBSC}}\right]-\mu}{\mu}\right|,
$$

where $\bar{Y}_{\text {LBSC }}$ is the statistical estimator under the LBSC approach. Note that $\mu$ is not known and needs to be estimated. To this end, we use an unbiased estimator based on 10,000,000 simulations, namely the crude MonteCarlo with the arithmetic lower bounds as control variates (CMC with ALBs).

We also compare the results with two other methods that were recently proposed in the literature. In Dingec and Hormann (2012), the authors propose a Monte Carlo method with control variate for the pricing of arithmetic Asian options in a Lévy setting. The idea of this method is to use as a control variate the geometric average Asian option payoff as calculated under the hypothesis of a geometric Brownian motion. The method is further based on the numerical inversion of the cumulative distribution function of the increments and is therefore applicable to Lévy processes for which the transition law is known. In Fusai and Meucci (2008), the authors propose a Monte Carlo method in which the control variate is the corresponding geometric Asian option but now computed in the (true) Lévy setting. The theoretical value of the geometric Asian option is here obtained using the Fast Fourier Transform method. We denote the methods of Fusai and Meucci (2008) and Dingec and Hormann (2012) in this paper further as CMC-GA_Lévy resp. CMC-GA_BS.

Finally, we make some remarks regarding the reported computation time (C). All methods require some pre-processing. In particular, the regression coefficients that are inherent in the approaches that use control variates need to be computed separately (to avoid bias). All these coefficients are computed from the same sample using 100000 replications. The computation time for estimating these coefficients are not included in the reported computation time results. Furthermore, the method of Fusai and Meucci (2008) uses the Fast Fourier Transform approach to price the value of the geometric Asian option that is used as control variate. Setting up this framework is 
not completely straightforward and the method needs to be adapted (i.e., the level of discretization and/or truncation used) to the specificities (e.g. the strike) of the payoff at hand. We do not consider these efforts when reporting computation time. Finally, the computational effort that is needed to estimate the bias of the LBSC approach is not included in the reported computation time for this method. Indeed, this computational effort only arises becasue it is not possible to estimate bias with a closed-form formula and does not reveal information on the intrinsic quality (speed) of the method itself.

\subsection{Numerical results for a Variance Gamma economy}

We first calculate Asian option prices in a VG model. We refer to equation (19) for the risk neutral price process and we take the parameters as in Table 8 of Albrecher et al. (2008), namely $\sigma=0.2684, \nu=1.1737, \theta=-0.1280$. This setting leads to an annual volatility for the log-returns of $30,211 \%$ (see also Dingec and Hormann (2012) for more details). In our numerical example we take as starting value $S_{0}=100$, as risk-free interest rate $r=0.03$ and we consider that the options are averaged monthly with maturity $T=10$ years, namely the number of averaging $n=120$ (see Table 1 ). We also give results for higher and lower volatility cases, namely, $\sigma=0.5884$ and $\sigma=0.058$ corresponding to annual volatilities of $60,452 \%$ and $15,031 \%$ respectively (see Table 2 and 3 respectively).

In Table 1, 2 and 3, the Mean, Rel. Bias, S.E., C, Eff-RF and Var-RF are presented in these VG settings for the different approaches and for different strikes. We also present the "True Value" which is estimated by using the CMC with ALB method and an extremely large number of simulations (10 millions).

\subsection{Numerical results for a Normal Inverse Gaussian econ- omy}

In this subsection we calculate Asian option prices in the settings of a NIG model. In Table 4, results are presented for Asian option prices in the settings of a NIG process, with the log-return following the density (20) and the parameters' values are taken from Albrecher and Predota (2004), namely $\alpha=136.29, \eta=-8.95, \delta=0.0059, \mu=0.00079$. The initial value $S_{0}$ is chosen to be 50 , the maturity is 20 days and the number of averaging days $n$ equals the number of days until maturity, namely $n=20$. The yearly riskfree interest rate equals $r=0.1$. We also give results for a higher volatility, namely $\delta=0.1059$, keeping the short maturity of 20 days (see Table 5). Finally, we present results for a high volatility, namely $\delta=0.5$ and a long maturity of 5 years with monthly averaging, $n=60$ (see Table 6 ). The 


\begin{tabular}{|c|c|c|c|c|c|c|c|c|}
\hline \multicolumn{9}{|c|}{ VG Model with an annual volatility of $30.211 \%$ and a maturity of 10 years } \\
\hline Strike & True Value & Approach & Mean & Rel. Bias & S.E. & $\mathrm{C}$. & Eff-RF & Var-RF \\
\hline \multirow{6}{*}{$K=60$} & \multirow{6}{*}{43.6440} & CMC & 43.7069 & - & 0.2043 & 3.09 & 1.0 & 1.0 \\
\hline & & CMC-GA_BS & 43.7912 & - & 0.1547 & 8.99 & 0.6 & 1.7 \\
\hline & & CMC-GA_Lévy & 43.6111 & - & 0.0263 & 4.10 & 45.5 & 60.3 \\
\hline & & CMC with ALBs & 43.6194 & - & 0.0184 & 3.80 & 100.2 & 123.3 \\
\hline & & CMC with GLBs & 43.5956 & - & 0.0318 & 3.74 & 34.1 & 41.3 \\
\hline & & LBSC & 43.6163 & $0.0633 \%$ & 0.0019 & 50.30 & 3.3 & 11561.9 \\
\hline \multirow{6}{*}{$K=80$} & \multirow{6}{*}{32.1815} & $\mathrm{CMC}$ & 32.2381 & - & 0.1918 & 3.07 & 1.0 & 1.0 \\
\hline & & CMC-GA_BS & 32.3366 & - & 0.1470 & 9.04 & 0.6 & 1.7 \\
\hline & & CMC-GA_Lévy & 32.1553 & - & 0.0243 & 4.04 & 47.3 & 62.3 \\
\hline & & CMC with ALBs & 32.1567 & - & 0.0179 & 3.77 & 93.5 & 114.8 \\
\hline & & CMC with GLBs & 32.1318 & - & 0.0290 & 3.71 & 36.2 & 43.7 \\
\hline & & LBSC & 32.1815 & $0.0357 \%$ & 0.0018 & 50.40 & 16.6 & 11354.1 \\
\hline \multirow{6}{*}{$K=100$} & \multirow{6}{*}{23.1446} & CMC & 23.1672 & - & 0.1747 & 3.09 & 1.0 & 1.0 \\
\hline & & CMC-GA_BS & 23.2488 & - & 0.1372 & 9.21 & 0.5 & 1.6 \\
\hline & & CMC-GA_Lévy & 23.1437 & - & 0.0226 & 4.16 & 44.4 & 59.8 \\
\hline & & CMC with ALBs & 23.1186 & - & 0.0170 & 3.70 & 88.2 & 105.6 \\
\hline & & CMC with GLBs & 23.0971 & - & 0.0253 & 3.62 & 40.7 & 47.7 \\
\hline & & LBSC & 23.1136 & $0.1339 \%$ & 0.0015 & 50.62 & 1.9 & 13564.5 \\
\hline \multirow{6}{*}{$\mathrm{K}=120$} & \multirow{6}{*}{16.4151} & CMC & 16.3973 & - & 0.1558 & 3.08 & 1.0 & 1.0 \\
\hline & & CMC-GA_BS & 16.4650 & - & 0.1257 & 9.21 & 0.5 & 1.5 \\
\hline & & CMC-GA_Lévy & 16.4400 & - & 0.0213 & 4.16 & 39.6 & 53.5 \\
\hline & & CMC with ALBs & 16.3942 & - & 0.0161 & 3.72 & 77.5 & 93.6 \\
\hline & & CMC with GLBs & 16.3790 & - & 0.0212 & 3.63 & 45.8 & 54.0 \\
\hline & & LBSC & 16.3870 & $0.1710 \%$ & 0.0010 & 50.20 & 1.9 & 24273.6 \\
\hline \multirow{6}{*}{$K=150$} & \multirow{6}{*}{9.7445} & CMC & 9.6845 & - & 0.1282 & 3.08 & 1.0 & 1.0 \\
\hline & & CMC-GA_BS & 9.7259 & - & 0.1077 & 9.01 & 0.5 & 1.4 \\
\hline & & CMC-GA_Lévy & 9.7753 & - & 0.0203 & 4.13 & 29.7 & 39.9 \\
\hline & & CMC with ALBs & 9.7265 & - & 0.0154 & 3.47 & 61.5 & 69.3 \\
\hline & & CMC with GLBs & 9.7214 & - & 0.0169 & 3.43 & 51.7 & 57.5 \\
\hline & & LBSC & 9.7152 & $0.1084 \%$ & 0.0007 & 50.60 & 8.9 & 33541.3 \\
\hline
\end{tabular}

Table 1: Summary of the simulations for VG Models with $\sigma=0.2684, \nu=$ 1.1737, $\theta=-0.1280, S_{0}=100, r=0.03$, the maturity is 10 years $(T=10)$ and there is monthly averaging $(\mathrm{n}=120)$. 


\begin{tabular}{|c|c|c|c|c|c|c|c|c|}
\hline \multicolumn{9}{|c|}{ VG Model with an annual volatility of $60.452 \%$ and a maturity of 10 years } \\
\hline Strike & True Value & Approach & Mean & Rel. Bias & S.E. & C. & Eff-RF & Var-RF \\
\hline \multirow{6}{*}{$\mathrm{K}=60$} & \multirow{6}{*}{50.5757} & CMC & 49.4934 & - & 0.8367 & 3.24 & 1.0 & 1.0 \\
\hline & & CMC-GA_BS & 49.3145 & - & 0.7660 & 9.18 & 0.4 & 1.2 \\
\hline & & CMC-GA_Lévy & 50.1774 & - & 0.3715 & 4.09 & 4.0 & 5.1 \\
\hline & & CMC with ALBs & 50.3151 & - & 0.1160 & 3.96 & 42.6 & 52.0 \\
\hline & & CMC with GLBs & 49.8362 & - & 0.4730 & 3.85 & 2.6 & 3.1 \\
\hline & & LBSC & 50.1856 & $0.7712 \%$ & 0.0056 & 51.07 & 0.3 & 22323.6 \\
\hline \multirow{6}{*}{$K=80$} & \multirow{6}{*}{43.8048} & CMC & 42.6894 & - & 0.8279 & 3.26 & 1.0 & 1.0 \\
\hline & & CMC-GA_BS & 42.5279 & - & 0.7628 & 9.30 & 0.4 & 1.2 \\
\hline & & CMC-GA_Lévy & 43.4574 & - & 0.3698 & 4.19 & 3.9 & 5.0 \\
\hline & & CMC with ALBs & 43.5767 & - & 0.1077 & 3.77 & 51.1 & 59.1 \\
\hline & & CMC with GLBs & 43.1395 & - & 0.4590 & 3.67 & 2.9 & 3.3 \\
\hline & & LBSC & 43.4394 & $0.8341 \%$ & 0.0107 & 50.64 & 0.3 & 5986.7 \\
\hline \multirow{6}{*}{$K=100$} & \multirow{6}{*}{38.7141} & CMC & 37.5352 & - & 0.8185 & 3.24 & 1.0 & 1.0 \\
\hline & & CMC-GA_BS & 37.4047 & - & 0.7589 & 9.13 & 0.4 & 1.2 \\
\hline & & CMC-GA_Lévy & 38.3246 & - & 0.3688 & 4.19 & 3.8 & 4.9 \\
\hline & & CMC with ALBs & 38.4763 & - & 0.1001 & 3.93 & 55.1 & 66.9 \\
\hline & & CMC with GLBs & 38.0813 & - & 0.4444 & 3.86 & 2.8 & 3.4 \\
\hline & & LBSC & 38.3370 & $0.9741 \%$ & 0.0110 & 50.15 & 0.3 & 5536.7 \\
\hline \multirow{6}{*}{$\mathrm{K}=120$} & \multirow{6}{*}{34.6617} & $\mathrm{CMC}$ & 33.5189 & - & 0.8091 & 3.26 & 1.0 & 1.0 \\
\hline & & CMC-GA_BS & 33.4197 & - & 0.7547 & 9.26 & 0.4 & 1.1 \\
\hline & & CMC-GA_Lévy & 34.3530 & - & 0.3685 & 4.22 & 3.7 & 4.8 \\
\hline & & CMC with ALBs & 34.4984 & - & 0.0933 & 3.74 & 65.6 & 75.2 \\
\hline & & CMC with GLBs & 34.0747 & - & 0.4297 & 4.09 & 2.8 & 3.5 \\
\hline & & LBSC & 34.3474 & $0.9067 \%$ & 0.0112 & 50.80 & 0.4 & 5218.8 \\
\hline \multirow{6}{*}{$\mathrm{K}=150$} & \multirow{6}{*}{30.0585} & $\mathrm{CMC}$ & 28.9148 & - & 0.7954 & 3.27 & 1.0 & 1.0 \\
\hline & & CMC-GA_BS & 28.8438 & - & 0.7479 & 9.17 & 0.4 & 1.1 \\
\hline & & CMC-GA_Lévy & 29.8106 & - & 0.3693 & 4.22 & 3.6 & 4.6 \\
\hline & & CMC with ALBs & 29.9484 & - & 0.0848 & 3.68 & 78.2 & 88.0 \\
\hline & & CMC with GLBs & 29.5825 & - & 0.4081 & 3.38 & 3.7 & 3.8 \\
\hline & & LBSC & 29.7612 & $0.9892 \%$ & 0.0103 & 51.00 & 0.5 & 5963.4 \\
\hline
\end{tabular}

Table 2: Summary of the simulations for VG Models with $\sigma=0.5884, \nu=$ 1.1737, $\theta=-0.1280, S_{0}=100, r=0.03$, the maturity is 10 years $(T=10)$ and there is monthly averaging $(\mathrm{n}=120)$. 


\begin{tabular}{|c|c|c|c|c|c|c|c|c|}
\hline \multicolumn{9}{|c|}{ VG Model with an annual volatility of $15.031 \%$ and a maturity of 10 years } \\
\hline Strike & True Value & Approach & Mean & Rel. Bias & S.E. & C. & Eff-RF & Var-RF \\
\hline \multirow{6}{*}{$\mathrm{K}=60$} & \multirow{6}{*}{42.1930} & CMC & 42.2141 & - & 0.0964 & 3.05 & 1.0 & 1.0 \\
\hline & & CMC-GA_BS & 42.2457 & - & 0.0794 & 9.06 & 0.5 & 1.5 \\
\hline & & CMC-GA_Lévy & 42.1844 & - & 0.0055 & 4.02 & 233.1 & 307.2 \\
\hline & & CMC with ALBs & 42.1899 & - & 0.0053 & 3.85 & 262.1 & 330.8 \\
\hline & & CMC with GLBs & 42.1869 & - & 0.0077 & 3.55 & 134.7 & 156.7 \\
\hline & & LBSC & 42.1876 & $0.0127 \%$ & 0.0064 & 51.15 & 8.0 & 226.9 \\
\hline \multirow{6}{*}{$K=80$} & \multirow{6}{*}{28.2478} & CMC & 28.2458 & - & 0.0903 & 3.03 & 1.0 & 1.0 \\
\hline & & CMC-GA_BS & 28.2773 & - & 0.0750 & 9.18 & 0.5 & 1.4 \\
\hline & & CMC-GA_Lévy & 28.2397 & - & 0.0047 & 4.01 & 278.9 & 369.1 \\
\hline & & CMC with ALBs & 28.2340 & - & 0.0068 & 3.74 & 142.9 & 176.3 \\
\hline & & CMC with GLBs & 28.2321 & - & 0.0082 & 3.54 & 103.8 & 121.3 \\
\hline & & LBSC & 28.2377 & $0.0357 \%$ & 0.0066 & 50.65 & 3.4 & 187.2 \\
\hline \multirow{6}{*}{$K=100$} & \multirow{6}{*}{16.3258} & CMC & 16.3192 & - & 0.0757 & 3.04 & 1.0 & 1.0 \\
\hline & & CMC-GA_BS & 16.3471 & - & 0.0642 & 9.34 & 0.5 & 1.4 \\
\hline & & CMC-GA_Lévy & 16.3300 & - & 0.0038 & 4.10 & 294.2 & 396.8 \\
\hline & & CMC with ALBs & 16.3292 & - & 0.0076 & 3.92 & 76.9 & 99.2 \\
\hline & & CMC with GLBs & 16.3285 & - & 0.0082 & 3.87 & 66.9 & 85.2 \\
\hline & & LBSC & 16.3204 & $0.0333 \%$ & 0.0061 & 50.33 & 5.2 & 153.5 \\
\hline \multirow{6}{*}{$\mathrm{K}=120$} & \multirow{6}{*}{7.7350} & CMC & 7.7266 & - & 0.0540 & 3.05 & 1.0 & 1.0 \\
\hline & & CMC-GA_BS & 7.7447 & - & 0.0473 & 9.61 & 0.4 & 1.3 \\
\hline & & CMC-GA_Lévy & 7.7528 & - & 0.0033 & 4.12 & 198.2 & 267.8 \\
\hline & & CMC with ALBs & 7.7311 & - & 0.0073 & 3.86 & 43.2 & 54.7 \\
\hline & & CMC with GLBs & 7.7301 & - & 0.0072 & 3.60 & 47.7 & 56.3 \\
\hline & & LBSC & 7.7306 & $0.0568 \%$ & 0.0050 & 51.10 & 3.9 & 116.6 \\
\hline \multirow{6}{*}{$\mathrm{K}=150$} & \multirow{6}{*}{1.4991} & CMC & 1.4876 & - & 0.0221 & 3.04 & 1.0 & 1.0 \\
\hline & & CMC-GA_BS & 1.4937 & - & 0.0204 & 9.39 & 0.4 & 1.2 \\
\hline & & CMC-GA_Lévy & 1.5112 & - & 0.0032 & 4.13 & 35.1 & 47.7 \\
\hline & & CMC with ALBs & 1.5056 & - & 0.0049 & 3.92 & 15.8 & 20.3 \\
\hline & & CMC with GLBs & 1.5088 & - & 0.0057 & 3.62 & 12.6 & 15.0 \\
\hline & & LBSC & 1.5007 & $0.1084 \%$ & 0.0034 & 50.40 & 2.1 & 42.3 \\
\hline
\end{tabular}

Table 3: Summary of the simulations for VG Models with $\sigma=0.058, \nu=$ $1.1737, \theta=-0.1280, S_{0}=100, r=0.03$, the maturity is 10 years $(T=10)$ and there is monthly averaging $(\mathrm{n}=120)$. 


\begin{tabular}{|c|c|c|c|c|c|c|c|c|}
\hline \multicolumn{9}{|c|}{ NIG Model with an annual volatility of $12.611 \%$ and a maturity of 20 days } \\
\hline Strike & True Value & Approach & Mean & Rel. Bias & S.E. & C. & Eff-RF & Var-RF \\
\hline \multirow{6}{*}{$\mathrm{K}=47$} & \multirow{6}{*}{3.1271} & CMC & 3.1248 & & 0.003959 & 0.48 & 1.0 & 1.0 \\
\hline & & CMC-GA_BS & 3.1271 & & 0.000788 & 1.07 & 11.3 & 25.2 \\
\hline & & CMC-GA_Lévy & 3.1270 & & 0.000017 & 1.00 & 27357.7 & 56995.3 \\
\hline & & CMC with ALBs & 3.1271 & & 0.000034 & 0.68 & 9776.0 & 13849.3 \\
\hline & & CMC with GLBs & 3.1271 & & 0.000038 & 0.70 & 7255.4 & 10580.8 \\
\hline & & LBSC & 3.1271 & $0.0001 \%$ & 0.000002 & 14.80 & 34667.5 & 3207703.5 \\
\hline \multirow{6}{*}{$\mathrm{K}=48.5$} & \multirow{6}{*}{1.6484} & $\mathrm{CMC}$ & 1.6466 & & 0.003821 & 0.50 & 1.0 & 1.0 \\
\hline & & CMC-GA_BS & 1.6485 & & 0.000738 & 1.08 & 12.4 & 26.8 \\
\hline & & CMC-GA_Lévy & 1.6492 & & 0.000015 & 1.02 & 33277.2 & 67885.5 \\
\hline & & CMC with ALBs & 1.6487 & & 0.000194 & 0.69 & 280.1 & 386.6 \\
\hline & & CMC with GLBs & 1.6487 & & 0.000196 & 0.72 & 264.3 & 380.6 \\
\hline & & LBSC & 1.6484 & $0.0006 \%$ & 0.000010 & 15.20 & 2548.1 & 161081.9 \\
\hline \multirow{6}{*}{$K=50$} & \multirow{6}{*}{0.4249} & $\mathrm{CMC}$ & 0.4241 & & 0.002524 & 0.49 & 1.0 & 1.0 \\
\hline & & CMC-GA_BS & 0.4247 & & 0.000526 & 1.08 & 10.5 & 23.1 \\
\hline & & CMC-GA_Lévy & 0.4250 & & 0.000011 & 1.05 & 25742.6 & 55162.6 \\
\hline & & CMC with ALBs & 0.4246 & & 0.000306 & 0.71 & 47.0 & 68.2 \\
\hline & & CMC with GLBs & 0.4246 & & 0.000306 & 0.69 & 48.3 & 68.0 \\
\hline & & LBSC & 0.4250 & $0.0017 \%$ & 0.000009 & 15.10 & 1494.2 & 73918.4 \\
\hline \multirow{6}{*}{$K=51.5$} & \multirow{6}{*}{0.0258} & CMC & 0.0256 & & 0.000632 & 0.53 & 1.0 & 1.0 \\
\hline & & CMC-GA_BS & 0.0253 & & 0.000231 & 1.11 & 3.6 & 7.4 \\
\hline & & CMC-GA_Lévy & 0.0280 & & 0.000008 & 1.09 & 3206.2 & 6593.8 \\
\hline & & CMC with ALBs & 0.0257 & & 0.000176 & 0.75 & 9.1 & 12.8 \\
\hline & & CMC with GLBs & 0.0257 & & 0.000177 & 0.76 & 8.9 & 12.7 \\
\hline & & LBSC & 0.0258 & $0.0021 \%$ & 0.000009 & 15.00 & 180.4 & 5125.9 \\
\hline
\end{tabular}

Table 4: Summary of the simulations for NIG Models with $\alpha=136.29$, $\eta=-8.95, \delta=0.0059, \mu=0.00079, S_{0}=50, r=0.1$, the maturity is 20 days $(T=20 / 365)$ and there is daily averaging $(\mathrm{n}=20)$.

performances of the different methods are summarized for different strikes.

\subsection{Discussion}

From an analysis of the Tables 1 to 6 , we observe that the LBSC approach is performing very well in terms of the variance reduction. It is in many cases the approach with the highest values for the variance reduction factor Var-RF (Tables 1, 2, 4, 5 and 6). In particular, the approach is performing outstanding when the volatility of the underlying risky asset reaches average to high levels. Recall that all other things being equal, the higher the variance reduction the smaller the number of simulations that are needed to obtain a given accuracy. Therefore, for a given acceptable bound on the standard error (S.E.), the LBSC approach often needs the smallest calculation time (even if we consider the fact that for each replication the method requires more running resources). We explain the feature of the high variance reduction ability for the LBSC as follows: The approach is based on conditioning, which allows to avoid simulation of the Brownian motion. More precisely, for each realization of the stochastic clock one uses an analytical formula for approximating the (conditional) option price. Next, by generating different scenarios for the clock one obtains an estimated price for the 


\begin{tabular}{|c|c|c|c|c|c|c|c|c|}
\hline \multicolumn{9}{|c|}{ NIG Model with an annual volatility of $53.428 \%$ and a maturity of 20 days } \\
\hline Strike & True Value & Approach & Mean & Rel. Bias & S.E. & C. & Eff-RF & Var-RF \\
\hline \multirow{6}{*}{$\mathrm{K}=47$} & \multirow{6}{*}{3.5060} & $\mathrm{CMC}$ & 3.5012 & & 0.014344 & 0.48 & 1.0 & 1.0 \\
\hline & & CMC-GA_BS & 3.5064 & & 0.000365 & 1.07 & 691.1 & 1540.6 \\
\hline & & CMC-GA_Lévy & 3.5069 & & 0.000204 & 1.00 & 2378.0 & 4954.3 \\
\hline & & CMC with ALBs & 3.5073 & & 0.001231 & 0.68 & 95.8 & 135.7 \\
\hline & & CMC with GLBs & 3.5073 & & 0.001258 & 0.70 & 89.1 & 129.9 \\
\hline & & LBSC & 3.5059 & $0.0030 \%$ & 0.000006 & 14.80 & 601.0 & 5079044.3 \\
\hline \multirow{6}{*}{$\mathrm{K}=48.5$} & \multirow{6}{*}{2.4177} & CMC & 2.4169 & & 0.012600 & 0.50 & 1.0 & 1.0 \\
\hline & & CMC-GA_BS & 2.4180 & & 0.000339 & 1.08 & 638.3 & 1378.7 \\
\hline & & CMC-GA_Lévy & 2.4188 & & 0.000187 & 1.02 & 2221.7 & 4532.3 \\
\hline & & CMC with ALBs & 2.4197 & & 0.001291 & 0.69 & 69.0 & 95.3 \\
\hline & & CMC with GLBs & 2.4198 & & 0.001311 & 0.72 & 64.2 & 92.4 \\
\hline & & LBSC & 2.4176 & $0.0045 \%$ & 0.000001 & 14.90 & 450.0 & 113211803.8 \\
\hline \multirow{6}{*}{$\mathrm{K}=50$} & \multirow{6}{*}{1.5549} & $\mathrm{CMC}$ & 1.5542 & & 0.010476 & 0.49 & 1.0 & 1.0 \\
\hline & & CMC-GA_BS & 1.5554 & & 0.000311 & 1.08 & 514.6 & 1134.3 \\
\hline & & CMC-GA_Lévy & 1.5553 & & 0.000174 & 1.05 & 1689.1 & 3619.4 \\
\hline & & CMC with ALBs & 1.5551 & & 0.001272 & 0.71 & 46.8 & 67.8 \\
\hline & & CMC with GLBs & 1.5552 & & 0.001281 & 0.69 & 47.5 & 66.9 \\
\hline & & LBSC & 1.5550 & $0.0060 \%$ & 0.000002 & 15.00 & 411.7 & 20992432.5 \\
\hline \multirow{6}{*}{$\mathrm{K}=51.5$} & \multirow{6}{*}{0.9288} & CMC & 0.9267 & & 0.008248 & 0.53 & 1.0 & 1.0 \\
\hline & & CMC-GA_BS & 0.9292 & & 0.000278 & 1.11 & 420.2 & 880.1 \\
\hline & & CMC-GA_Lévy & 0.9311 & & 0.000163 & 1.09 & 1244.4 & 2559.1 \\
\hline & & CMC with ALBs & 0.9276 & & 0.001197 & 0.75 & 33.6 & 47.5 \\
\hline & & CMC with GLBs & 0.9276 & & 0.001197 & 0.76 & 33.1 & 47.5 \\
\hline & & LBSC & 0.9289 & $0.0113 \%$ & 0.000001 & 15.10 & 216.7 & 32759400.1 \\
\hline
\end{tabular}

Table 5: Summary of the simulations for NIG Models with $\alpha=136.29$, $\eta=-8.95, \delta=0.1059, \mu=0.00079, S_{0}=50, r=0.1$, the maturity is 20 days $(T=20 / 365)$ and there is daily averaging $(n=20)$. 


\begin{tabular}{|c|c|c|c|c|c|c|c|c|}
\hline \multicolumn{9}{|c|}{ NIG Model with an annual volatility of $21.05 \%$ and a maturity of 5 years } \\
\hline Strike & True Value & Approach & Mean & Rel. Bias & S.E. & C. & Eff-RF & Var-RF \\
\hline \multirow{6}{*}{$\mathrm{K}=45$} & \multirow{6}{*}{12.6220} & CMC & 12.6258 & & 0.050729 & 1.12 & 1.0 & 1.0 \\
\hline & & CMC-GA_BS & 12.6403 & & 0.004346 & 1.82 & 83.8 & 136.2 \\
\hline & & CMC-GA_Lévy & 12.6238 & & 0.004364 & 2.00 & 75.7 & 135.1 \\
\hline & & CMC with ALBs & 12.6234 & & 0.003498 & 1.54 & 153.0 & 210.3 \\
\hline & & CMC with GLBs & 12.6238 & & 0.005214 & 1.88 & 56.4 & 94.7 \\
\hline & & LBSC & 12.6191 & $0.0232 \%$ & 0.000004 & 31.20 & 10.8 & 130013302.8 \\
\hline \multirow{6}{*}{$K=50$} & \multirow{6}{*}{10.1101} & CMC & 10.1218 & & 0.048370 & 1.14 & 1.0 & 1.0 \\
\hline & & CMC-GA_BS & 10.1292 & & 0.004246 & 1.85 & 80.0 & 129.8 \\
\hline & & CMC-GA_Lévy & 10.1141 & & 0.004254 & 2.20 & 67.0 & 129.3 \\
\hline & & CMC with ALBs & 10.1110 & & 0.003777 & 1.54 & 121.4 & 164.0 \\
\hline & & CMC with GLBs & 10.1113 & & 0.005139 & 1.82 & 55.5 & 88.6 \\
\hline & & LBSC & 10.1075 & $0.0255 \%$ & 0.000004 & 32.20 & 12.5 & 142851816.1 \\
\hline \multirow{6}{*}{$\mathrm{K}=55$} & \multirow{6}{*}{7.9216} & $\mathrm{CMC}$ & 7.9205 & & 0.045000 & 1.12 & 1.0 & 1.0 \\
\hline & & CMC-GA_BS & 7.9329 & & 0.004065 & 1.82 & 75.4 & 122.5 \\
\hline & & CMC-GA_Lévy & 7.9192 & & 0.004067 & 2.33 & 58.8 & 122.4 \\
\hline & & CMC with ALBs & 7.9207 & & 0.003865 & 1.55 & 98.0 & 135.6 \\
\hline & & CMC with GLBs & 7.9201 & & 0.004743 & 1.89 & 53.3 & 90.0 \\
\hline & & LBSC & 7.9193 & $0.0288 \%$ & 0.000003 & 31.50 & 13.8 & 177883174.4 \\
\hline \multirow{6}{*}{$K=60$} & \multirow{6}{*}{6.0872} & $\mathrm{CMC}$ & 6.1020 & & 0.041040 & 1.13 & 1.0 & 1.0 \\
\hline & & CMC-GA_BS & 6.0955 & & 0.003918 & 1.84 & 67.4 & 109.7 \\
\hline & & CMC-GA_Lévy & 6.0835 & & 0.003910 & 2.29 & 54.4 & 110.2 \\
\hline & & CMC with ALBs & 6.0835 & & 0.003822 & 1.56 & 83.5 & 115.3 \\
\hline & & CMC with GLBs & 6.0815 & & 0.004264 & 1.91 & 54.8 & 92.6 \\
\hline & & LBSC & 6.0852 & $0.0323 \%$ & 0.000003 & 31.30 & 15.7 & 220145773.8 \\
\hline
\end{tabular}

Table 6: Summary of the simulations for NIG Models with $\alpha=136.29$, $\eta=-8.95, \delta=0.5, \mu=0.00079 * 30, S_{0}=50, r=0.1$, the maturity is 5 years $(T=5)$ and there is monthly averaging $(\mathrm{n}=60)$. 
(unconditional) option price. It is then clear that the variance of the estimator using the LBSC approach is lower than the estimator when using the traditional Monte Carlo simulation. Intuitively, this difference in variance is expected to be quite significant because the stochastic clock is only one source of randomness for the Asian option price, and is independent of the second source, which is the Brownian motion. Unfortunately, and mainly driven by the presence of bias, this efficiency in terms of variance reduction is not reflected in the other efficiency measure Eff-Rf. One also observes that the bias is increasing in maturity and volatility but remains rather small. We believe that the LBSC approach is particularly useful in a risk management context because the feature of variance reduction may be considered as more important than the fact that the approach provides estimates that are biased. Note indeed that especially in risk management computation time is still an issue because simulations are typically carried out in the real world and the risk-neutral world jointly (e.g. when calculating the risk for a portfolio of options over a one year horizon one needs to compute prices in the risk neutral world conditional on the occurrence of real-world scenarios). In this instance, small pricing errors can surely be neglected but speed is important. Therefore, for Asset-Liability management purposes, the LBSC approach is a very suitable candidate.

As for the unbiased Monte Carlo approaches that are based on control variates, the analysis of the different tables shows that there are two approaches that depart from the others and show nice performance statistics. These are the crude Monte-Carlo with the arithmetic lower bounds as control variates (CMC with ALBs) and the control variate approach (CMC-GA_Lévy) of Fusai and Meucci (2008). Tables 1, 2 and 6 show that the CMC with ALBs method is outperforming for the different criteria considered whereas Tables 3, 4 and 5 support the use of CMC-GA_Lévy. A closer inspection of the specifications of the different numerical illustrations, in particular considering the aspects maturity and volatility, suggests that the method of Fusai and Meucci (2008) is better adapted to deal with products that have a rather short maturity (Tables 4 and 5) or when the volatility of the underlying is rather low (Table 3). In contrast, the CMC with ALBs approach is outperforming when the maturities involved are longer (like in insurance products) and in these instances its performance increases with increasing volatility; see Tables 1, 2 and 6 . We further investigate these observations by performing a further sensitivity analysis of the factors maturity and volatility using a NIG economy. The results of this analysis are shown in Table 7 and 8 and confirm the above findings. In summary, the numerical examples provide strong evidence that the CMC with ALBs approach is suitable in an insurance context that involves longer maturities, whereas the method of Fusai and Meucci (2008) is more suitable in a trading context involving shorter maturities. On a more qualitative note, we point out that the CMC with ALBs approach and the CMC with GLBs approach computes the ex- 


\begin{tabular}{|c|c|c|c|c|c|c|}
\hline \multicolumn{7}{|c|}{ NIG Model with an annual volatility of $30.07 \%$ and a maturity of 1 year } \\
\hline Strike & Approach & Mean & S.E. & $\mathrm{C}$. & Eff-RF & Var-RF \\
\hline \multirow{3}{*}{$\mathrm{K}=45$} & CMC & 7.8910 & 0.0354 & 0.23 & 1.0 & 1.0 \\
\hline & CMC-GA_Lévy & 7.9164 & 0.0014 & 0.37 & 399.6 & 642.8 \\
\hline & CMC with ALBs & 7.9119 & 0.0030 & 0.22 & 149.4 & 142.9 \\
\hline \multirow{3}{*}{$\mathrm{K}=60$} & $\mathrm{CMC}$ & 1.3985 & 0.0172 & 0.25 & 1.0 & 1.0 \\
\hline & CMC-GA_Lévy & 1.4057 & 0.0012 & 0.40 & 137.0 & 219.2 \\
\hline & CMC with ALBs & 1.4054 & 0.0027 & 0.26 & 38.8 & 40.3 \\
\hline \multicolumn{7}{|c|}{ NIG Model with an annual volatility of $30.07 \%$ and a maturity of 5 years } \\
\hline Strike & Approach & Mean & S.E. & C. & Eff-RF & Var-RF \\
\hline \multirow{3}{*}{$\mathrm{K}=45$} & CMC & 13.4823 & 0.0720 & 1.00 & 1.0 & 1.0 \\
\hline & CMC-GA_Lévy & 13.4578 & 0.0081 & 1.70 & 46.8 & 79.5 \\
\hline & CMC with ALBs & 13.4667 & 0.0059 & 1.15 & 129.3 & 148.7 \\
\hline \multirow{3}{*}{$\mathrm{K}=60$} & $\mathrm{CMC}$ & 7.8520 & 0.0615 & 1.10 & 1.0 & 1.0 \\
\hline & CMC-GA_Lévy & 7.8560 & 0.0075 & 1.83 & 40.3 & 67.0 \\
\hline & CMC with ALBs & 7.8518 & 0.0057 & 1.32 & 98.5 & 118.1 \\
\hline \multicolumn{7}{|c|}{ NIG Model with an annual volatility of $30.07 \%$ and a maturity of 10 years } \\
\hline Strike & Approach & Mean & S.E. & C. & Eff-RF & Var-RF \\
\hline \multirow{3}{*}{$\mathrm{K}=45$} & CMC & 16.0506 & 0.0972 & 5.60 & 1.0 & 1.0 \\
\hline & CMC-GA_Lévy & 16.0415 & 0.0200 & 8.90 & 14.8 & 23.5 \\
\hline & CMC with ALBs & 16.0302 & 0.0088 & 6.72 & 102.3 & 122.7 \\
\hline \multirow{3}{*}{$\mathrm{K}=60$} & $\mathrm{CMC}$ & 12.2261 & 0.0923 & 5.60 & 1.0 & 1.0 \\
\hline & CMC-GA_Lévy & 12.2298 & 0.0193 & 9.05 & 14.2 & 22.9 \\
\hline & CMC with ALBs & 12.2151 & 0.0083 & 6.65 & 104.2 & 123.8 \\
\hline
\end{tabular}

Table 7: Summary of the simulations for NIG Models with $\alpha=136.29$, $\eta=-8.95, \mu=0.00079, \delta=1.02, S_{0}=50, r=0.1$, the maturity are 1,5 and 10 years and there is monthly averaging.

pectations of the control variates using Gauss Laguerre integration whereas the method of Fusai and Meucci (2008) requires the Fast Fourier Transform approach. We found the former approach rather easy to implement and did not encounter major difficulties. The implementation of the Fast Fourier Transform requires a careful handling of discretization and truncation (see also Carr and Madan (1998)), which makes it sometimes more difficult to use.

\section{Applications to other products}

\subsection{Unit Linked Insurance}

Unit linked insurance products are life type insurance products where policyholder's premiums are used to purchase units in investment funds generally 


\begin{tabular}{|c|c|c|c|c|c|c|}
\hline \multicolumn{7}{|c|}{ NIG Model with an annual volatility of $40.05 \%$ and a maturity of 1 year } \\
\hline Strike & Approach & Mean & S.E. & $\mathrm{C}$. & Eff-RF & Var-RF \\
\hline \multirow{3}{*}{$\mathrm{K}=45$} & CMC & 8.6853 & 0.0461 & 0.25 & 1.0 & 1.0 \\
\hline & CMC-GA_Lévy & 8.7163 & 0.0025 & 0.40 & 218.8 & 350.1 \\
\hline & CMC with ALBs & 8.7129 & 0.0042 & 0.27 & 113.8 & 122.9 \\
\hline \multirow{3}{*}{$\mathrm{K}=60$} & CMC & 2.4488 & 0.0277 & 0.27 & 1.0 & 1.0 \\
\hline & CMC-GA_Lévy & 2.4598 & 0.0021 & 0.44 & 102.4 & 166.9 \\
\hline & CMC with ALBs & 2.4586 & 0.0038 & 0.30 & 47.2 & 52.5 \\
\hline \multicolumn{7}{|c|}{ NIG Model with an annual volatility of $40.05 \%$ and a maturity of 5 years } \\
\hline Strike & Approach & Mean & S.E. & $\mathrm{C}$. & Eff-RF & Var-RF \\
\hline \multirow{3}{*}{$\mathrm{K}=45$} & CMC & 14.6706 & 0.0996 & 1.10 & 1.0 & 1.0 \\
\hline & CMC-GA_Lévy & 14.6663 & 0.0147 & 1.80 & 28.2 & 46.2 \\
\hline & CMC with ALBs & 14.6692 & 0.0089 & 1.30 & 105.4 & 124.6 \\
\hline \multirow{3}{*}{$\mathrm{K}=60$} & CMC & 9.7760 & 0.0890 & 1.15 & 1.0 & 1.0 \\
\hline & CMC-GA_Lévy & 9.7992 & 0.0138 & 1.88 & 25.4 & 41.5 \\
\hline & CMC with ALBs & 9.7969 & 0.0081 & 1.35 & 103.7 & 121.7 \\
\hline \multicolumn{7}{|c|}{ NIG Model with an annual volatility of $40.05 \%$ and a maturity of 10 years } \\
\hline Strike & Approach & Mean & S.E. & C. & Eff-RF & Var-RF \\
\hline \multirow{3}{*}{$\mathrm{K}=45$} & CMC & 17.0460 & 0.1473 & 6.00 & 1.0 & 1.0 \\
\hline & CMC-GA_Lévy & 17.0598 & 0.0448 & 9.20 & 7.0 & 10.8 \\
\hline & CMC with ALBs & 17.0398 & 0.0172 & 7.00 & 62.6 & 73.0 \\
\hline \multirow{3}{*}{$K=60$} & $\mathrm{CMC}$ & 13.8717 & 0.1423 & 5.88 & 1.0 & 1.0 \\
\hline & CMC-GA_Lévy & 13.8974 & 0.0437 & 9.14 & 6.8 & 10.6 \\
\hline & CMC with ALBs & 13.8764 & 0.0160 & 6.86 & 67.9 & 79.2 \\
\hline
\end{tabular}

Table 8: Summary of the simulations for NIG Models with $\alpha=136.29$, $\eta=-8.95, \mu=0.00079, \delta=1.81, S_{0}=50, r=0.1$, the maturity are 1,5 and 10 years and there is monthly averaging. 
composed of different assets such as for example bonds and stocks, usually selected by the policyholder ${ }^{8}$. Since the fund value $\left(S_{t}\right)_{t}$ is composed of risky assets, the insurance company generally proposes a minimum guarantee at maturity in order to protect the policyholder against an economy downfall. Let assume that the policyholder invests only in a single investment fund or stock and that investment premiums $P_{i}$ are paid at time $t_{i}, i=0,1,2, \ldots, n-1$ which allow the policyholder to purchase $P_{i} / S_{t_{i}}$ units and each unit has a value $S_{T}$ at expiry $T=t_{n}$. The payoff of the contract at maturity $T$ (conditional upon survival of the insured until time $T$ ) is therefore given by the maximum of the fund value $\left(F V_{n}\right)$ and a certain minimum payment $K$

$$
\max \left(F V_{n}, K\right)=\max \left(\sum_{i=0}^{n-1} P_{i} \frac{S_{T}}{S_{t_{i}}}, K\right)=K+\max \left(\sum_{i=0}^{n-1} P_{i} \frac{S_{T}}{S_{t_{i}}}-K, 0\right) .
$$

For example in the case of a guaranteed rate of return $R$, insurance companies often determine $K$ to be equal to $K=\sum_{i=0}^{n-1} P_{i} e^{R\left(T-t_{i}\right)}$ (see Schrager and Pelsser (2004)) where the investment premiums $P_{i}$ can be a function of the guaranteed rate of return $R$.

Following Schrager and Pelsser (2004), we assume that at time $i=0, \ldots, n-1$ the premium $P_{i}$ is given by $P_{i}=G P_{i}-F C_{i}-c_{i} F V_{i}$, where $G P_{i}$ is the gross premium at time $i$ which is paid at regular intervals until expiry of the insurance contract; $F C_{i}$ is a fixed cost at time $i$, including investment costs, administration costs; $c_{i}$ is the fund value related cost deduction (including mortality charges) and $F V_{i}$ is the fund value at time $i$. In Schrager and Pelsser (2004), it is shown that

$$
\sum_{i=0}^{n-1} P_{i} \frac{S_{T}}{S_{t_{i}}}=\sum_{i=0}^{n-1} \widetilde{P}_{i}^{n} \frac{S_{T}}{S_{t_{i}}}
$$

where $\widetilde{P}_{i}^{n}=\left(G P_{i}-F C_{i}\right) \prod_{j=1}^{n-i-1}\left(1-c_{n-j}\right)$ which is deterministic. In particular if $c_{i}$ is constant over time and denoted by $c_{i} \equiv c$ we have $\widetilde{P}_{i}^{n}=$ $\left(G P_{i}-F C_{i}\right)(1-c)^{n-i-1}$. In that case, the no-arbitrage price of the unit linked contract can be rewritten as

$$
P=K+E\left[\max \left(\sum_{i=0}^{n-1} \widetilde{P}_{i}^{n} \frac{S_{T}}{S_{t_{i}}}-K, 0\right)\right] .
$$

Following our approach of modelling the spot price by an exponential VG or NIG process, namely $S_{t}=S_{0} e^{Z_{t}}$ where $Z_{t}$ is a VG or NIG process, and using the independent and stationary increment properties of Lévy processes, we obtain

$$
\sum_{i=0}^{n-1} \widetilde{P}_{i}^{n} \frac{S_{T}}{S_{t_{i}}}=\sum_{i=0}^{n-1} \widetilde{P}_{i}^{n} e^{Z_{T}-Z_{t_{i}}} \stackrel{\mathcal{D}}{=} \sum_{i=0}^{n-1} \widetilde{P}_{i}^{n} e^{Z_{T-t_{i}}^{\prime}}
$$

\footnotetext{
${ }^{8}$ Some insurers even give the possibility to invest in individual stocks (see Schrager and Pelsser (2004))
} 
where $Z^{\prime}$ is an independent copy of the Lévy process $Z$, and where $\stackrel{\mathcal{D}}{=}$ reflects equality in law.

The no-arbitrage price of the unit linked contract is now given by

$$
P=K+E\left[\max \left(\sum_{i=0}^{n-1} \widetilde{P}_{i}^{n} e^{\left.Z_{T-t_{i}}^{\prime}-K, 0\right)}\right] .\right.
$$

It is easy to observe the relation between unit linked insurance products and Asian options and therefore the approaches developed in this paper are suitable to price such insurance contracts.

\subsection{Ratchet equity-indexed annuities (EIAs)}

In this subsection, we focus on pricing ratchet equity-indexed annuities (EIAs) with Asian-end design. An equity-indexed annuity contract is a fixed annuity earning a minimum rate of interest and offering a potential gain which is tied to the performance of an underlying equity index $S$ which is in general measured on the basis of the returns generated by the index over the lifetime of the contract. There exist different types of EIAs and the most popular ones are the annual ratchet EIAs. In the case of annual ratchet EIAs, the index return level $R_{t}$ is measured each year based on the equity index performances. In the literature you can find different designs for $R_{t}$ and according to Lin and Tan (2003), the most common type of design is based on averaging (which according to Marrion $(2000,1)$ represent approximately $60 \%$ of Annual Reset EIAs), for example the index return is often determined by $R_{t}=\frac{1}{N} \frac{\sum_{i=0}^{N-1} S_{t-i / N}}{S_{t-1}}-1$, where $N$ is the number of reset dates in one year to calculate the index return measure.

According to Lin and Tan (2003) and Marrion (2000,1), ratchet EIAs are among the most popular contracts in the North America market in terms of their sales volume. According to Marrion (2000,1), $70 \%$ of EIAs sold in the market are of this type. There exist two types of ratchet EIAs, namely the simple ratchet EIAs and the compounded ratchet EIAs. Following Ballotta (2010), simple ratchet EIAs payoffs $B_{T}^{(s)}$ and compounded ratchet EIAs payoffs $B_{T}^{(c)}$ at maturity $T$ are respectively given by

$$
\begin{aligned}
& B_{T}^{(s)}=1+T F+\sum_{t=1}^{T}\left[\left(\alpha R_{t}-F\right)^{+}-\left(\alpha R_{t}-C\right)^{+}\right] \\
& B_{T}^{(c)}=\prod_{t=1}^{T}\left[1+F+\left(\alpha R_{t}-F\right)^{+}-\left(\alpha R_{t}-C\right)^{+}\right]
\end{aligned}
$$

where $C$ and $F \in(0,1)$ denote fixed Cap and Floor rates respectively providing respectively an upper bound to the rate of return and a minimum guarantee, and where $R_{t}$ reflects the index return and $\alpha$ a fixed percentage, 
called the participation rate.

Therefore, ratchet EIAs can be seen as a portfolio composed of a risk-free component and a sequence of bull spreads composed of call options of types depending on the design of $R_{t}$. Using the moment-matching method and assuming a VG economy for the equity index $S_{t}$, Ballotta (2010) has derived an approximation for a ratchet EIA where the index return is measured by $R_{t}=\frac{1}{N} \frac{\sum_{i=0}^{N-1} S_{t-i / N}}{S_{t-1}}-1$, where $N$ is the number of reset dates in one year to calculate the index level measure.

Modelling the equity index $S$ by an exponential VG or NIG process, namely $S_{t}=S_{0} e^{Z_{t}}$ where $Z_{t}$ is a VG or NIG process (see Section 4 ), and because of independent and stationary increment properties of Lévy processes, the no-arbitrage price of the ratchet EIAs is in the settings of Ballotta (2010) in the simple case given by

$$
\begin{aligned}
B_{0}^{(s)}=e^{-r T}(1+T F) & +e^{-r T} E\left[T\left(\frac{\alpha}{N} \sum_{i=0}^{N-1} e^{Z_{1-i / N}^{\prime}}-K_{F}\right)^{+}\right] \\
& -e^{-r T} E\left[T\left(\frac{\alpha}{N} \sum_{i=0}^{N-1} e^{Z_{1-i / N}^{\prime}}-K_{C}\right)^{+}\right]
\end{aligned}
$$

and in the compounded case by

$B_{0}^{(c)}=e^{-r T} E\left[1+F+\left(\frac{\alpha}{N} \sum_{i=0}^{N-1} e^{Z_{1-i / N}^{\prime}}-K_{F}\right)^{+}-\left(\frac{\alpha}{N} \sum_{i=0}^{N-1} e^{Z_{1-i / N}^{\prime}}-K_{C}\right)^{+}\right]^{T}$

where $K_{F}=\alpha+F$ and $K_{C}=\alpha+C$; and where as before $Z^{\prime}$ is an independent copy of the Lévy process $Z$. Hence the pricing problem of such ratchet EIAs with Asian-end design reduces to the problem of pricing two Asian options with a maturity of one year and a strike of $K_{F}$ and $K_{C}$. It is now easy to understand that the approximating price approaches developed in this paper to price Asian options can be useful for insurance companies that deal with ratchet EIAs.

\section{Conclusions}

In this paper we propose two methods for pricing Asian style payoffs in a Lévy market setting. A first approach consists in using model-independent lower bounds for Asian options as control variates in a traditional Monte Carlo scheme (CMC with ALBs approach). Numerical results show that this approach outperforms other (unbiased) methods when the contracts involve longer maturities. In contrast, the Monte Carlo method with geometric control variate of Fusai and Meucci (2008) seems to be more efficient in a rather short maturity context. Note that the CMC with ALBs approach avoids the use of FFT (needed in the well known Fusai and Meucci (2008) 
method), which requires more programming efforts and needs to be adjusted to the specificities of the payoff at hand (issues of discretization, truncation and singularities). In this respect Mont Carlo methods are more general approaches that often require less programming efforts than deterministic numerical techniques. We conclude that the (unbiased) CMC with ALBs approach is suitable in a pricing context especially for the pricing of insurance Asian type derivatives as these involve longer maturities. Note that we also briefly discussed the application of the methods presented in this paper to other insurance products like ratchet equity-indexed annuities and unit linked insurance agreements.

A second approach (LBSC) is based on a conditioning on the stochastic clock, which makes the nature of the problem multi-variate normal and allows us to make use of very accurate closed form approximations that we apply conditionally. Next, Monte-Carlo simulations with control variates are used to account for the stochasticity of the clock itself. This approach is biased but the bias appears to be small in practical examples. Moreover, the method is simple to implement and its variance reduction ability usually surpasses all the other methods developed in this paper as well as the popular control variate Monte Carlo methods of Fusai and Meucci (2008) and Dingec and Hormann (2012). Therefore, the last method offers an attractive tradeoff between accuracy and efficiency (speed), which is particularly useful in a risk management context.

\section{Acknowledgments}

The authors would like to thank the anonymous referees for their constructive suggestions and comments. Griselda Deelstra acknowledges support of the ARC grant IAPAS "Interaction between Analysis, Probability and Actuarial Sciences" 2012-2017. Steven Vanduffel acknowledges the financial support of the BNP Paribas Fortis Chair in Banking. Grgory Raye is supported by a Mandat de Charg de Recherche from the Fonds National de la Recherche Scientifique, Communaut franaise de Belgique.

\section{References}

M. Abramowitz and C. A. Stegun. Handbook of Mathematical Functions with Formulas, Graphs and Mathematical Tables. Dover, (1964).

H. Albrecher, Ph. Mayer, and W. Schoutens. General lower bounds for arithmetic asian option prices. Applied Mathematical Finance, 15:123149, (2008).

H. Albrecher and M. Predota. On asian option pricing for NIG lévy pro- 
cesses. Journal of Computational and applied mathemaics, 172:153-168, (2004).

L. Bachelier. Théorie de la spéculation. PhD thesis, Gauthier-Villars (Paris), (1900).

L. Ballotta. Efficient pricing of ratchet equity-indexed annuities in a variance-gamma economy. North American Actuarial Journal, 14:355$368,(2010)$.

O. E. Barndorff-Nielsen. Processes of normal inverse gaussian type. Finance and Stochastics, 2:41-68, (1998).

P. Boyle and A. Potapchik. Prices and sensitivities of asian options: A survey. Insurance: Mathematics and Economics, 42:189-211, (2008).

S. Browne, M. A. Milevsky, and T. S. Salisbury. Asset allocation and the liquidity premium for illiquid annuities. Journal of Risk and Insurance, 70:509-226, (2003).

P. Carr and A. Chou. Breaking barriers. Risk, 10:139-145, (1997).

P. Carr, H. Geman, D. Madan, and M. Yor. The fine structure of asset returns: An empirical investigation. Journal of Business, 75:305-332, (2002).

P. Carr and D. Madan. Option valuation using the fast fourier transform. Journal of Computational Finance, 2:61-73, (1998).

R. Cont and P. Tankov. Financial modeling with jump processes. Chapman \& Hall/Crc Financial Mathematics Series, (2004).

K.D. Dingec and W. Hormann. A general control variate method for option pricing under lévy process. European Journal of Operational Research, 221:368-377, (2012).

E. Eberlein and U. Keller. Hyperbolic distributions in finance. Bernoulli, 1: 281-299, (1995).

E. Eberlein, U. Keller, and K. Prause. New insights into smile, mispricing and value at risk: the hyperbolic model. Journal of Business, 71:371-405, (1998).

L.F. Fenton. The sum of log-normal probability distributions in scatter transmission systems. IEEE Transactions on Communication Systems, 8: 57-67, (1960).

M. Fu, D. Madan, and T. Wang. Pricing continuous asian options: a comparison of monte carlo and laplace transform inversion methods. Journal of Computational Finance, 2:49-74, (1999). 
G. Fusai and A. Meucci. Pricing discretely monitored asian option under lévy processes. Journal of Banking and Finance, 32:2076-2088, (2008).

J. Garcia, S. Goosens, M. Viktoriya, and W. Schoutens. Lévy base correlation. Technical report 07-04, section of statistics, k.u.leuven, (2007).

H. Gerber and S.W. Shiu. Option Pricing by Esscher Transforms. Transactions of the Society of Actuaries, 46:99-191, (1994).

P. Glasserman. Monte Carlo Methods in Financial Engineering. Springer, (2003).

R. Kaas, J. Dhaene, and M. Goovaerts. Upper and lower bounds for sums of random variables. Insurance: Mathematics and Economics, 27:151-168, (2000).

R. Kaas, M. Goovaerts, J. Dhaene, and M. Denuit. Modern actuarial risk theory. Springer, (2008).

A. Kemna and A. Vorst. A pricing method for options based on average asset values. Journal of Banking and Finance, 14:113-130, (1990).

P. L'Ecuyer. Efficiency improvement and variance reduction. In Simulation Conference Proceedings, (1994).

X. Lin and K. Tan. Valuation of equity-indexed annuities under stochastic interest rates. North American Actuarial Journal, 7:72-93, (2003).

D. Madan, P. Carr, and E. Chang. The variance gamma process and option pricing. European Finance Review, 2:79-105, (1998).

D. Madan and E. Senata. The variance gamma model for share market returns. Journal of Business, 63:511-524, (1990).

D.B. Madan, M.R. Pistorius, and W. Schoutens. The valuation of structured products using markov chain models. Quantitative Finance, 13:125-136, (2011).

B. Mandelbrot. The variation of certain speculative prices. The Journal of Business, 36:394-419, (1963).

J. Marrion. Advantage 2000 equity index report, (november) online at http://www.indexannuity.org., (2000).

J. Marrion. Advantage 2001 equity index report, (march) online at http://www.indexannuity.org., (2001).

M. Milevsky and S. Posner. The titanic option: Valuation of the guaranteed minimum death benefit in variable annuities and mutual funds. The Journal of Risk and Insurance, 68:93-128, (2003). 
I. Monroe. Processes that can be embedded in brownian motion. The Annals of Probability, 6:42-56, (1978).

A. Müller and D. Stoyan. Comparison Methods for Stochastic Models and Risks. Wiley \& Sons, Inc., (2002).

K. Prause. The generalized hyperbolic model: estimation, financial derivatives, and risk measures. PhD thesis, University of Freiburg, (1999).

W. H. Press, S. A. Teukolsky, W. T. Vetterling, and B. P. Flannery. Numerical Recipes in C. Cambridge University Press, (1992).

M. Romeo, V. Da Costa, and F. Bardou. Broad distribution effects in sums of lognormal random variables. The European Physical Journal B, 32: $513-525,(2003)$.

K. Sato. Lévy Processes and Infinitely Divisible Distributions. Cambridge University Press, (1999).

W. Schoutens. Lévy Processes in Finance:Pricing Financial Derivatives. Wiley, (2003).

D. Schrager and A. Pelsser. Pricing rate of return guarantees in regular premium unit linked insurance. Insurance: Mathematics and Economics, 35:369-398, (2004).

M. Shaked and J. G. Shanthikumar. Stochastic Orders and Their Applications. Academic Press, New York, (1994).

A. H. Stroud and D. Secrest. Gaussian Quadrature Formulas. Prentice-Hal, (1966).

E.A. Valdez, J. Dhaene, M. Maj, and S. Vanduffel. Bounds and approximations for sums of dependent log-elliptical random variables. Insurance: Mathematics and Economics, 44(3):385-397, (2009).

S. Vanduffel, X. Chen, J. Dhaene, M. Goovaerts, L. Henrard, and R. Kaas. Optimal approximations for risk measure of sums of lognormals based on conditional expectations. Journal of Computational and Applied Mathematics, 221:202-218, (2008a).

S. Vanduffel, Z. Shang, L. Henrard, J. Dhaene, and E.A. Valdez. Analytic bounds and approximations for annuities and asian options. Insurance: Mathematics and Economics, 42(3):1109-1117, (2008b). 